Supporting Information for

\title{
Stepwise Cope Rearrangement of Cyclo-Biphenalenyl via an Unusual Multicenter Covalent $\pi$-Bonded Intermediate
}

\author{
Jingsong Huang and Miklos Kertesz* \\ Department of Chemistry, Georgetown University, $37^{\text {th }}$ \& O Street, Washington, DC 20057-1227
}

\section{Author lists truncated:}

(11a) Goto, K.; Kubo, T.; Yamamoto, K.; Nakasuji, K.; Sato, K.; Shiomi, D.; Takui, T.; Kubota, M.; Kobayashi, T.; Takusi, K.; Ouyang, J. Y. J. Am. Chem. Soc. 1999, 121, 1619.

(26) Frisch, M. J.; Trucks, G. W.; Schlegel, H. B.; Scuseria, G. E.; Robb, M. A.; Cheeseman, J. R.; Zakrzewski, V. G.; Montgomery, J. A., Jr.; Stratmann, R. E.; Burant, J. C.; Dapprich, S.; Millam, J. M.; Daniels, A. D.; Kudin, K. N.; Strain, M. C.; Farkas, O.; Tomasi, J.; Barone, V.; Cossi, M.; Cammi, R.; Mennucci, B.; Pomelli, C.; Adamo, C.; Clifford, S.; Ochterski, J.; Petersson, G. A.; Ayala, P. Y.; Cui, Q.; Morokuma, K.; Malick, D. K.; Rabuck, A. D.; Raghavachari, K.; Foresman, J. B.; Cioslowski, J.; Ortiz, J. V.; Stefanov, B. B.; Liu, G.; Liashenko, A.; Piskorz, P.; Komaromi, I.; Gomperts, R.; Martin, R. L.; Fox, D. J.; Keith, T.; Al-Laham, M. A.; Peng, C. Y.; Nanayakkara, A.; Gonzalez, C.; Challacombe, M.; Gill, P. M. W.; Johnson, B. G.; Chen, W.; Wong, M. W.; Andres, J. L.; Head-Gordon, M.; Replogle, E. S.; Pople, J. A.

(42) Morita, Y.; Aoki, T.; Fukui, K.; Nakazawa, S.; Tamaki, K.; Suzuki, S.; Fuyuhiro, A.; Yamamoto, K.; Sato, K.; Shiomi, D.; Naito, A.; Takui, T.; Nakasuji, K. Angew. Chem., Int. Ed. 2002, 41, 1793.

(44) Kubo, T.; Shimizu, A.; Sakamoto, M.; Uruichi, M.; Yakushi, K.; Nakano, M.; Shiomi, D.; Sato, K.; Takui, T.; Morita, Y.; Nakasuji, K. Angew. Chem., Int. Ed. 2005, 44,6564 . 


\section{Chart 1S.}
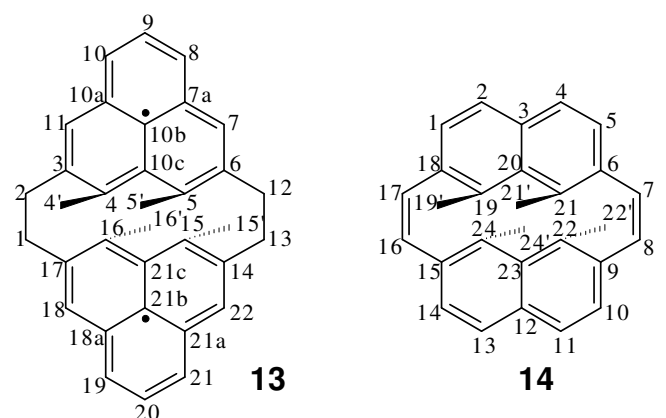

Table 1S. Calculated geometries of the $\pi$-bonded structures and the $\sigma$-bonded structure of $\mathbf{1 3}$ found by R(U)B3LYP. For atomic numbering, see Chart $1 \mathrm{~S}$. For the $\mathrm{C}_{2 h}$ symmetry only the non-redundant information is listed.

\begin{tabular}{|c|c|c|c|}
\hline Index & $\begin{array}{c}13 \\
\pi \text {-bonded structure } \\
\end{array}$ & $\begin{array}{c}13 \\
\pi \text {-bonded structure } \\
\end{array}$ & $\begin{array}{c}13 \\
\sigma \text {-bonded structure }\end{array}$ \\
\hline Notes & RB3LYP & UB3LYP & R(U)B3LYP \\
\hline Symmetry $^{b}$ & $\mathrm{C}_{2 h}$ & $\mathrm{C}_{2 h}$ & $\mathrm{C}_{2}$ \\
\hline $\mathrm{C} 1-\mathrm{C} 2$ & 1.575 & 1.574 & 1.564 \\
\hline $\mathrm{C} 2-\mathrm{C} 3$ & 1.521 & 1.523 & 1.515 \\
\hline $\mathrm{C} 3-\mathrm{C} 4$ & 1.433 & 1.423 & 1.520 \\
\hline C4-C4' & 1.513 & 1.515 & 1.551 \\
\hline C4-C10c & 1.446 & 1.448 & 1.538 \\
\hline C3-C11 & 1.379 & 1.388 & 1.346 \\
\hline C10a-C11 & 1.421 & 1.414 & 1.448 \\
\hline $\mathrm{C} 10 \mathrm{a}-\mathrm{C} 10 \mathrm{~b}$ & 1.431 & 1.431 & 1.434 \\
\hline C10b-C10c & 1.445 & 1.442 & 1.435 \\
\hline C10-C10a & 1.411 & 1.416 & 1.389 \\
\hline C9-C10 & 1.391 & 1.391 & 1.407 \\
\hline $\mathrm{C} 8-\mathrm{C} 9$ & & & 1.379 \\
\hline $\mathrm{C} 7 \mathrm{a}-\mathrm{C} 8$ & & & 1.418 \\
\hline $\mathrm{C} 7 \mathrm{a}-\mathrm{C} 10 \mathrm{~b}$ & & & 1.432 \\
\hline $\mathrm{C} 7-\mathrm{C} 7 \mathrm{a}$ & & & 1.417 \\
\hline C6-C7 & & & 1.376 \\
\hline $\mathrm{C} 5-\mathrm{C} 6$ & & & 1.442 \\
\hline C5-C10c & & & 1.412 \\
\hline $\mathrm{C} 5-\mathrm{C} 5$ & & & 1.520 \\
\hline C6-C12 & & & 1.521 \\
\hline C12-C13 & & & 1.579 \\
\hline $\mathrm{C} 4-\mathrm{C} 16\left(\mathrm{R}_{1}\right)$ & 2.905 & 3.261 & 1.733 \\
\hline $\mathrm{C} 5-\mathrm{C} 15\left(\mathrm{R}_{2}\right)$ & & & 2.783 \\
\hline
\end{tabular}

${ }^{\text {a }}$ Second-order saddle point; the two imaginary frequencies correspond to methyl rotations. ${ }^{\mathrm{b}}$ In case of $\mathrm{C}_{2 h}$ symmetry, $\mathrm{R}_{1}=\mathrm{R}_{2}$. 
Table 2S. Calculated geometries of the neutral 14, $\pi$-bonded structures of $14^{2+}, \pi$-bonded structures and the $\sigma$-bonded structure of $\mathbf{1 4}^{2-}$ found by R(U)B3LYP. For atomic numbering, see Chart $1 \mathrm{~S}$.

\begin{tabular}{|c|c|c|c|c|c|}
\hline Index & $\mathbf{1 4}$ & $\begin{array}{c}\mathbf{1 4}^{2+} \\
\pi \text {-bonded }\end{array}$ & $\begin{array}{c}\mathbf{1 4}^{2-} \\
\pi \text {-bonded }\end{array}$ & $\begin{array}{c}\mathbf{1 4}^{2-} \\
\pi \text {-bonded }\end{array}$ & $\begin{array}{c}\mathbf{1 4}^{2-} \\
\sigma \text {-bonded }\end{array}$ \\
\hline Notes & $\mathrm{R}(\mathrm{U}) \mathrm{B} 3 \mathrm{LYP}$ & $\mathrm{R}(\mathrm{U}) \mathrm{B} 3 \mathrm{~L} Y \mathrm{P}$ & $\mathrm{R}(\mathrm{U}) \mathrm{B} 3 \mathrm{LYP}$ & $\mathrm{R}(\mathrm{U}) \mathrm{B} 3 \mathrm{LYP}$ & $\mathrm{R}(\mathrm{U}) \mathrm{B} 3 \mathrm{LYP}$ \\
\hline Symmetry & $\mathrm{C}_{2 h}$ & $\mathrm{C}_{2 h}$ & $\mathrm{C}_{2 h}$ & $\mathrm{C}_{2}$ & $\mathrm{C}_{2}$ \\
\hline C1-C2 & 1.370 & 1.384 & 1.389 & 1.393 & 1.406 \\
\hline C2-C3 & 1.420 & 1.412 & 1.422 & 1.413 & 1.415 \\
\hline C1-C18 & 1.422 & 1.404 & 1.408 & 1.408 & 1.381 \\
\hline C3-C20 & 1.441 & 1.443 & 1.460 & 1.460 & 1.458 \\
\hline C18-C19 & 1.400 & 1.446 & 1.435 & 1.424 & 1.527 \\
\hline C19-C20 & 1.449 & 1.441 & 1.440 & 1.447 & 1.535 \\
\hline C17-C18 & 1.487 & 1.463 & 1.462 & 1.476 & 1.450 \\
\hline C16-C17 & 1.353 & 1.359 & 1.386 & 1.374 & 1.375 \\
\hline C19-C19' & 1.515 & 1.509 & 1.518 & 1.515 & 1.560 \\
\hline C3-C4 & & & & 1.430 & 1.427 \\
\hline C4-C5 & & & & 1.383 & 1.384 \\
\hline C5-C6 & & & & 1.413 & 1.407 \\
\hline C6-C7 & & & & 1.447 & 1.474 \\
\hline C7-C8 & & & & 1.400 & 1.372 \\
\hline C6-C21 & & & & 1.446 & 1.437 \\
\hline C20-C21 & & & & 1.533 & 1.414 \\
\hline C21-C21 & & & & 2.989 & 1.525 \\
\hline C19-C24 $\left(R_{1}\right)$ & 3.207 & 2.883 & & 3.008 & 2.726 \\
\hline C21-C22 $\left(R_{2}\right)$ & & & & & \\
\hline
\end{tabular}

${ }^{\mathrm{a}}$ Second-order saddle point. On the PES of $\mathbf{1 4}^{2-}$, this is flanked by two degenerate distorted $\mathrm{C}_{2}$ structures, one of which is shown in the following column.

${ }^{\mathrm{b}}$ In case of $\mathrm{C}_{2 h}$ symmetry, $\mathrm{R}_{1}=\mathrm{R}_{2}$.
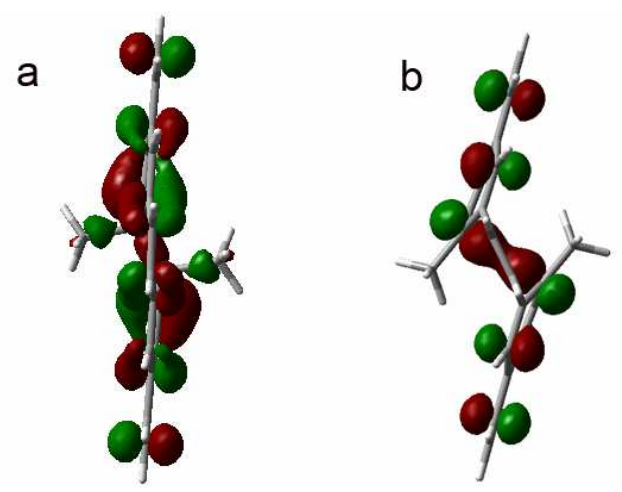

Figure 1S. Side views of the HOMOs for the aromatic intermediate 11 (a) and the $\pi$ intermediate $\mathbf{1 0}$ (b) calculated using RB3LYP/6-31G*, showing the planarity of $\mathbf{1 1}$ and the $\pi$-dimer character of $\mathbf{1 0}$. 


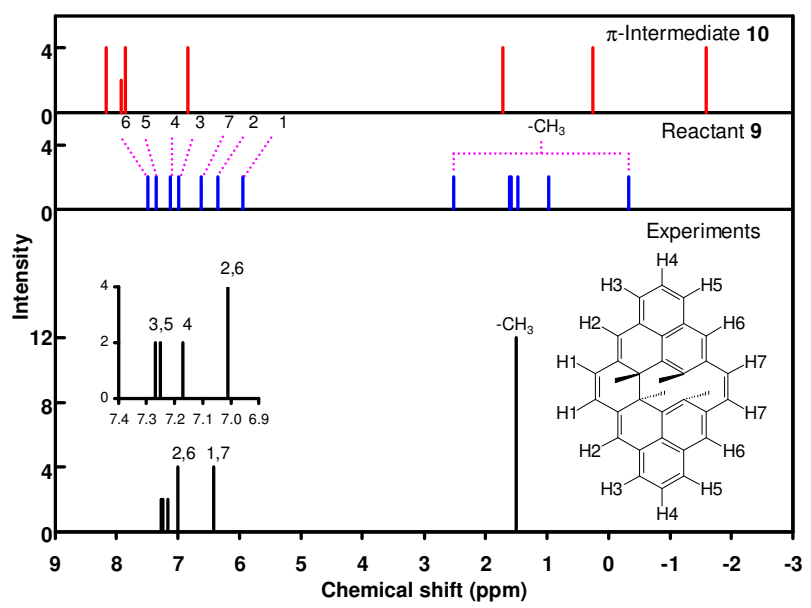

Figure 2S. Experimental ${ }^{1} \mathrm{H}$ NMR spectrum of 9 at RT from ref 23 compared with calculated peaks from B3LYP/6-311+G(2d) calculations with the B3LYP/6-311+G(2d) optimized geometries. Chemical shift is relative to the TMS value of $32.3443 \mathrm{ppm}$ from B3LYP/6-311+G(2d) calculation with the B3LYP/6-311+G(2d) optimized geometry.

Table 3S. Optimized geometries (as Cartesian coordinates) of all calculated structures. $\mathrm{R}(\mathrm{U}) \mathrm{B} 3 \mathrm{LYP} / 6-31 \mathrm{G}^{*}$ singlet of $\mathbf{9}, \mathrm{C}_{2}$ symmetry:

\begin{tabular}{|l|rrr|l|rrr|}
\hline & $\mathrm{x}$ & $\mathrm{y}$ & $\mathrm{z}$ & & $\mathrm{x}$ & $\mathrm{y}$ & $\mathrm{z}$ \\
\hline $\mathrm{C}$ & -0.217091 & 0.641343 & 3.748525 & $\mathrm{H}$ & -0.357876 & 1.177643 & 4.684816 \\
$\mathrm{C}$ & 0.217091 & -0.641343 & 3.748525 & $\mathrm{H}$ & 0.357876 & -1.177643 & 4.684816 \\
$\mathrm{C}$ & 0.459967 & -1.360419 & 2.501714 & $\mathrm{H}$ & 1.984783 & 1.271755 & 0.958467 \\
$\mathrm{C}$ & 0.676703 & -0.501272 & 1.255632 & $\mathrm{H}$ & 2.846000 & -0.255561 & 1.215739 \\
$\mathrm{C}$ & 1.971662 & 0.332512 & 1.510102 & $\mathrm{H}$ & 2.080903 & 0.572779 & 2.567393 \\
$\mathrm{C}$ & -0.676703 & 0.501272 & 1.255632 & $\mathrm{H}$ & -1.984783 & -1.271755 & 0.958467 \\
$\mathrm{C}$ & -1.971662 & -0.332512 & 1.510102 & $\mathrm{H}$ & -2.846000 & 0.255561 & 1.215739 \\
$\mathrm{C}$ & -0.459967 & 1.360419 & 2.501714 & $\mathrm{H}$ & -2.080903 & -0.572779 & 2.567393 \\
$\mathrm{C}$ & -0.402411 & 2.709836 & 2.452363 & $\mathrm{H}$ & -0.269657 & 3.274209 & 3.374563 \\
$\mathrm{C}$ & -0.383282 & 3.447211 & 1.209226 & $\mathrm{H}$ & -0.158559 & 5.359601 & 2.143257 \\
$\mathrm{C}$ & -0.223958 & 4.828436 & 1.196600 & $\mathrm{H}$ & 0.000000 & 6.620144 & 0.005035 \\
$\mathrm{C}$ & -0.131105 & 5.541700 & -0.013042 & $\mathrm{H}$ & -0.109631 & 5.404672 & -2.155749 \\
$\mathrm{C}$ & -0.196274 & 4.867878 & -1.214135 & $\mathrm{H}$ & 0.269657 & -3.274209 & 3.374563 \\
$\mathrm{C}$ & -0.381299 & 3.461387 & -1.248015 & $\mathrm{H}$ & -0.075518 & 3.235928 & -3.381088 \\
$\mathrm{C}$ & -0.487130 & 2.725444 & -0.025483 & $\mathrm{H}$ & 1.633475 & 1.410128 & -1.042545 \\
$\mathrm{C}$ & 0.791608 & -1.319903 & -0.038250 & $\mathrm{H}$ & 2.990623 & 0.285984 & -1.006087 \\
$\mathrm{C}$ & 0.402411 & -2.709836 & 2.452363 & $\mathrm{H}$ & 2.184385 & 0.646172 & -2.528020 \\
$\mathrm{C}$ & -0.791608 & 1.319903 & -0.038250 & $\mathrm{H}$ & 0.158559 & -5.359601 & 2.143257 \\
$\mathrm{C}$ & -0.375851 & 2.732702 & -2.464063 & $\mathrm{H}$ & -1.633475 & -1.410128 & -1.042545 \\
$\mathrm{C}$ & 1.123420 & -0.735485 & -1.273074 & $\mathrm{H}$ & -2.990623 & -0.285984 & -1.006087 \\
$\mathrm{C}$ & 0.487130 & -2.725444 & -0.025483 & $\mathrm{H}$ & -2.184385 & -0.646172 & -2.528020 \\
$\mathrm{C}$ & 0.383282 & -3.447211 & 1.209226 & $\mathrm{H}$ & -0.531033 & 1.055036 & -4.650249 \\
$\mathrm{C}$ & -1.123420 & 0.735485 & -1.273074 & $\mathrm{H}$ & 0.531033 & -1.055036 & -4.650249 \\
$\mathrm{C}$ & -0.677058 & 1.388064 & -2.481344 & $\mathrm{H}$ & 0.075518 & -3.235928 & -3.381088
\end{tabular}




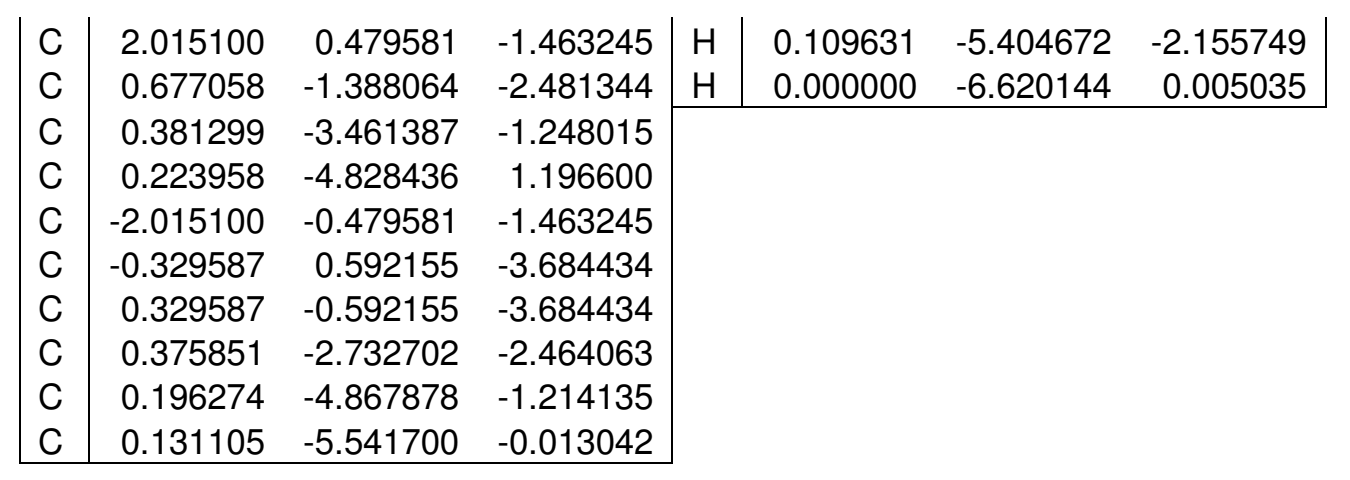

RB3LYP/6-311+G(2d) singlet of 9, $\mathrm{C}_{2}$ symmetry:

\begin{tabular}{|c|c|c|c|c|c|c|c|}
\hline & $x$ & $y$ & z & & $x$ & $y$ & z \\
\hline C & -0.170179 & 0.651606 & 3.737548 & $\mathrm{H}$ & -0.275202 & 1.192508 & 4.672879 \\
\hline C & 0.170179 & -0.651606 & 3.737548 & $\mathrm{H}$ & 0.275202 & -1.192508 & 4.672879 \\
\hline C & 0.359099 & -1.387435 & 2.495272 & $\mathrm{H}$ & 2.079347 & 1.105996 & 0.940134 \\
\hline C & 0.638045 & -0.550611 & 1.250921 & $\mathrm{H}$ & 2.814655 & -0.470098 & 1.226013 \\
\hline C & 1.986995 & 0.182919 & 1.502299 & $\mathrm{H}$ & 2.110633 & 0.430157 & 2.551961 \\
\hline C & -0.638045 & 0.550611 & 1.250921 & $\mathrm{H}$ & -2.079347 & -1.105996 & 0.940134 \\
\hline C & -1.986995 & -0.182919 & 1.502299 & $\mathrm{H}$ & -2.814655 & 0.470098 & 1.226013 \\
\hline C & -0.359099 & 1.387435 & 2.495272 & $\mathrm{H}$ & -2.110633 & -0.430157 & 2.551961 \\
\hline C & -0.195469 & 2.721781 & 2.446309 & $\mathrm{H}$ & -0.022263 & 3.271198 & 3.368154 \\
\hline C & -0.113399 & 3.454559 & 1.207135 & $\mathrm{H}$ & 0.253115 & 5.338804 & 2.139544 \\
\hline C & 0.151899 & 4.814375 & 1.195127 & $\mathrm{H}$ & 0.517478 & 6.578413 & 0.009246 \\
\hline C & 0.303410 & 5.515961 & -0.009633 & $\mathrm{H}$ & 0.321590 & 5.383307 & -2.144201 \\
\hline C & 0.190907 & 4.853339 & -1.206659 & $\mathrm{H}$ & 0.022263 & -3.271198 & 3.368154 \\
\hline C & -0.102020 & 3.469542 & -1.242982 & $\mathrm{H}$ & 0.191629 & 3.220440 & -3.369544 \\
\hline C & -0.270122 & 2.746912 & -0.024981 & $\mathrm{H}$ & 1.749274 & 1.269341 & -1.064939 \\
\hline C & 0.685485 & -1.374876 & -0.039982 & $\mathrm{H}$ & 2.999151 & 0.042326 & -0.994224 \\
\hline C & 0.195469 & -2.721781 & 2.446309 & $\mathrm{H}$ & 2.251504 & 0.443722 & -2.523724 \\
\hline C & -0.685485 & 1.374876 & -0.039982 & $\mathrm{H}$ & -0.253115 & -5.338804 & 2.139544 \\
\hline C & -0.151899 & 2.745281 & -2.455521 & $\mathrm{H}$ & -1.749274 & -1.269341 & -1.064939 \\
\hline C & 1.063392 & -0.825415 & -1.271595 & $\mathrm{H}$ & -2.999151 & 2326 & -0.994224 \\
\hline C & 0.270122 & -2.746912 & -0.024981 & $\mathrm{H}$ & -2.251504 & -0.443722 & -2.523724 \\
\hline C & 0.113399 & -3.454559 & 1.207135 & $\mathrm{H}$ & -0.444190 & 1.087741 & -4.637442 \\
\hline C & -1.063392 & 0.825415 & -1.271595 & $\mathrm{H}$ & 0.444190 & -1.087741 & -4.637442 \\
\hline C & -0.560591 & 1.435544 & -2.474524 & $\mathrm{H}$ & -0.191629 & -3.220440 & -3.369544 \\
\hline C & 2.051596 & 0.305552 & -1.464408 & $\mathrm{H}$ & -0.321590 & -5.383307 & -2.144201 \\
\hline C & 0.560591 & -1.435544 & -2.474524 & $\mathrm{H}$ & -0.517478 & -6.578413 & 0.009246 \\
\hline C & 0.102020 & -3.469542 & -1.242982 & & & & \\
\hline C & -0.151899 & -4.814375 & 1.195127 & & & & \\
\hline C & -2.051596 & -0.305552 & -1.464408 & & & & \\
\hline C & -0.277192 & 0.614216 & -3.673302 & & & & \\
\hline C & 0.277192 & -0.614216 & -3.673302 & & & & \\
\hline C & 0.151899 & -2.745281 & -2.455521 & & & & \\
\hline C & -0.190907 & -4.853339 & -1.206659 & & & & \\
\hline C & -0.303410 & -5.515961 & -0.009633 & & & & \\
\hline
\end{tabular}


Transition structure of 9 by R(U)B3LYP/6-31G*, $\mathrm{C}_{2}$ symmetry:

\begin{tabular}{|c|c|c|c|c|c|c|c|}
\hline & $\mathrm{x}$ & $\mathrm{y}$ & $z$ & & $\mathrm{x}$ & $y$ & $\mathrm{z}$ \\
\hline C & -0.294713 & 0.607651 & 3.711535 & $\mathrm{H}$ & -0.491606 & 1.106051 & 4.659012 \\
\hline C & 0.294713 & -0.607651 & 3.711535 & $\mathrm{H}$ & 0.491606 & -1.106051 & 4.659012 \\
\hline C & 0.607273 & -1.348137 & 2.477482 & $\mathrm{H}$ & 1.824093 & 1.417398 & 0.956693 \\
\hline C & 0.949839 & -0.590052 & 1.260920 & $\mathrm{H}$ & 2.988501 & 0.108552 & 1.191036 \\
\hline C & 2.006952 & 0.488532 & 1.496951 & $\mathrm{H}$ & 2.073284 & 0.731748 & 2.557051 \\
\hline C & -0.949839 & 0.590052 & 1.260920 & $\mathrm{H}$ & -1.824093 & -1.417398 & 0.956693 \\
\hline C & -2.006952 & -0.488532 & 1.496951 & $\mathrm{H}$ & -2.988501 & -0.108552 & 1.191036 \\
\hline C & -0.607273 & 1.348137 & 2.477482 & $\mathrm{H}$ & -2.073284 & -0.731748 & 2.557051 \\
\hline C & -0.436968 & 2.698370 & 2.448082 & $\mathrm{H}$ & -0.198979 & 3.232067 & 3.366611 \\
\hline C & -0.447129 & 3.441050 & 1.218288 & $\mathrm{H}$ & -0.154293 & 5.353796 & 2.146453 \\
\hline C & -0.250533 & 4.825061 & 1.201217 & $\mathrm{H}$ & 0.000000 & 6.601430 & 0.001558 \\
\hline C & -0.154261 & 5.525808 & -0.007175 & $\mathrm{H}$ & -0.122897 & 5.372568 & -2.152355 \\
\hline C & -0.230623 & 4.843281 & -1.208744 & $\mathrm{H}$ & 0.198979 & -3.232067 & 3.366611 \\
\hline C & -0.438267 & 3.444595 & -1.234488 & $\mathrm{H}$ & -0.107591 & 3.207814 & -3.362744 \\
\hline C & -0.580740 & 2.720457 & -0.010927 & $\mathrm{H}$ & 1.631218 & 1.460766 & -1.094802 \\
\hline C & 0.930489 & -1.316299 & -0.016303 & $\mathrm{H}$ & 3.015024 & 0.371788 & -1.005668 \\
\hline C & 0.436968 & -2.698370 & 2.448082 & $\mathrm{H}$ & 2.221771 & 0.669838 & -2.548101 \\
\hline C & -0.930489 & 1.316299 & -0.016303 & $\mathrm{H}$ & 0.154293 & -5.353796 & 2.146453 \\
\hline C & -0.415713 & 2.705439 & -2.447914 & $\mathrm{H}$ & -1.631218 & -1.460766 & -1.094802 \\
\hline C & 1.165409 & -0.695080 & -1.273740 & $\mathrm{H}$ & -3.015024 & -0.371788 & -1.005668 \\
\hline C & 0.580740 & -2.720457 & -0.010927 & $\mathrm{H}$ & -2.221771 & -0.669838 & -2.548101 \\
\hline C & 0.447129 & -3.441050 & 1.218288 & $\mathrm{H}$ & -0.555316 & 1.048872 & -4.640625 \\
\hline C & -1.165409 & 0.695080 & -1.273740 & $\mathrm{H}$ & 0.555316 & -1.048872 & -4.640625 \\
\hline C & -0.699715 & 1.358968 & -2.467459 & $\mathrm{H}$ & 591 & -3.207814 & -3.362744 \\
\hline C & 2.041946 & 0.525944 & -1.4 & $\mathrm{H}$ & 97 & -5.3 & 52355 \\
\hline C & 0.699715 & -1.35 & -2.467459 & $\mathrm{H}$ & 0.000000 & -6.601430 & 0.001558 \\
\hline C & 0.438267 & -3.444595 & -1.234488 & & & & \\
\hline C & 0.250533 & -4.825061 & 1.201217 & & & & \\
\hline C & -2.041946 & -0.525944 & -1.481428 & & & & \\
\hline C & -0.339831 & 0.584736 & -3.678651 & & & & \\
\hline C & 0.339831 & -0.584736 & -3.678651 & & & & \\
\hline C & 0.415713 & -2.705439 & -2.447914 & & & & \\
\hline C & 0.230623 & -4.843281 & -1.208744 & & & & \\
\hline C & 0.154261 & -5.525808 & -0.007175 & & & & \\
\hline
\end{tabular}

Transition structure of 9 by RB3LYP/6-311+G(2d), $C_{2}$ symmetry:

\begin{tabular}{|l|rrr|l|rrr|}
\hline & $\mathrm{x}$ & $\mathrm{y}$ & $\mathrm{z}$ & & $\mathrm{x}$ & $\mathrm{y}$ & $\mathrm{z}$ \\
\hline $\mathrm{C}$ & -0.235489 & 0.629097 & 3.702341 & $\mathrm{H}$ & -0.389461 & 1.140154 & 4.647894 \\
$\mathrm{C}$ & 0.235489 & -0.629097 & 3.702341 & $\mathrm{H}$ & 0.389461 & -1.140154 & 4.647894 \\
$\mathrm{C}$ & 0.479853 & -1.392443 & 2.471708 & $\mathrm{H}$ & 1.947121 & 1.240643 & 0.951682 \\
$\mathrm{C}$ & 0.880040 & -0.664756 & 1.255955 & $\mathrm{H}$ & 2.973056 & -0.167704 & 1.188170 \\
$\mathrm{C}$ & 2.035136 & 0.302273 & 1.490576 & $\mathrm{H}$ & 2.129312 & 0.540958 & 2.545169 \\
$\mathrm{C}$ & -0.880040 & 0.664756 & 1.255955 & $\mathrm{H}$ & -1.947121 & -1.240643 & 0.951682 \\
$\mathrm{C}$ & -2.035136 & -0.302273 & 1.490576 & $\mathrm{H}$ & -2.973056 & 0.167704 & 1.188170 \\
$\mathrm{C}$ & -0.479853 & 1.392443 & 2.471708 & $\mathrm{H}$ & -2.129312 & -0.540958 & 2.545169 \\
$\mathrm{C}$ & -0.200941 & 2.717477 & 2.442021 & $\mathrm{H}$ & 0.073655 & 3.228792 & 3.360450
\end{tabular}




\begin{tabular}{|c|c|c|c|c|c|c|c|}
\hline C & -0.141380 & 3.456885 & 1.215056 & $\mathrm{H}$ & 0.307686 & 5.332970 & 2.141216 \\
\hline C & 0.171562 & 4.813659 & 1.198481 & $\mathrm{H}$ & 0.576202 & 6.557365 & 0.004402 \\
\hline C & 0.331052 & 5.501413 & -0.005516 & $\mathrm{H}$ & 0.355056 & 5.351751 & -2.142392 \\
\hline C & 0.200941 & 4.832120 & -1.202687 & $\mathrm{H}$ & -0.073655 & -3.228792 & 3.360450 \\
\hline C & -0.125270 & 3.460124 & -1.230772 & $\mathrm{H}$ & 0.187653 & 3.194591 & -3.352763 \\
\hline C & -0.332670 & 2.753936 & -0.011578 & $\mathrm{H}$ & 1.768870 & 1.296777 & -1.101452 \\
\hline C & 0.801942 & -1.390459 & -0.018613 & $\mathrm{H}$ & 3.032838 & 0.086187 & -1.000107 \\
\hline C & 0.200941 & -2.717477 & 2.442021 & $\mathrm{H}$ & 2.287093 & 0.449261 & -2.540302 \\
\hline C & -0.801942 & 1.390459 & -0.018613 & $\mathrm{H}$ & -0.307686 & -5.332970 & 2.141216 \\
\hline C & -0.166322 & 2.724293 & -2.440264 & $\mathrm{H}$ & -1.768870 & -1.296777 & -1.101452 \\
\hline C & 1.099033 & -0.801724 & -1.271551 & $\mathrm{H}$ & -3.032838 & -0.086187 & -1.000107 \\
\hline C & 0.332670 & -2.753936 & -0.011578 & $\mathrm{H}$ & -2.287093 & -0.449261 & -2.540302 \\
\hline C & 0.141380 & -3.456885 & 1.215056 & $\mathrm{H}$ & -0.458369 & 1.087496 & -4.627900 \\
\hline C & -1.099033 & 0.801724 & -1.271551 & $\mathrm{H}$ & 0.458369 & -1.087496 & -4.627900 \\
\hline C & -0.571326 & 1.415063 & -2.460809 & $\mathrm{H}$ & -0.187653 & -3.194591 & -3.352763 \\
\hline C & 2.084366 & 0.326891 & -1.479395 & $\mathrm{H}$ & -0.355056 & -5.351751 & -2.142392 \\
\hline C & 0.571326 & -1.415063 & -2.460809 & $\mathrm{H}$ & -0.576202 & -6.557365 & 0.004402 \\
\hline C & 0.125270 & -3.460124 & -1.230772 & & & & \\
\hline C & -0.171562 & -4.813659 & 1.198481 & & & & \\
\hline C & -2.084366 & -0.326891 & -1.479395 & & & & \\
\hline C & -0.282368 & 0.610386 & -3.667297 & & & & \\
\hline C & 0.282368 & -0.610386 & -3.667297 & & & & \\
\hline C & 0.166322 & -2.724293 & -2.440264 & & & & \\
\hline C & -0.200941 & -4.832120 & -1.202687 & & & & \\
\hline & -0.331052 & -5.501413 & -0.005516 & & & & \\
\hline
\end{tabular}

RB3LYP/6-31G* singlet of 10, $\mathrm{C}_{2 h}$ symmetry:

\begin{tabular}{|l|rrr|l|rrr|}
\hline & \multicolumn{1}{|l|}{$\mathrm{x}$} & $\mathrm{y}$ & & $\mathrm{x}$ & $\mathrm{y}$ & $\mathrm{z}$ \\
\hline $\mathrm{C}$ & -0.296972 & 0.606464 & 3.660099 & $\mathrm{H}$ & -0.481242 & 1.085684 & 4.621206 \\
$\mathrm{C}$ & 0.296972 & -0.606464 & 3.660099 & $\mathrm{H}$ & 0.481242 & -1.085684 & 4.621206 \\
$\mathrm{C}$ & 0.601439 & -1.402699 & 2.447585 & $\mathrm{H}$ & 1.783998 & 1.310040 & 1.057753 \\
$\mathrm{C}$ & 1.163329 & -0.796317 & 1.272245 & $\mathrm{H}$ & 3.095173 & 0.129817 & 1.059981 \\
$\mathrm{C}$ & 2.114627 & 0.360922 & 1.488280 & $\mathrm{H}$ & 2.258684 & 0.525677 & 2.556975 \\
$\mathrm{C}$ & -1.163329 & 0.796317 & 1.272245 & $\mathrm{H}$ & -1.783998 & -1.310040 & 1.057753 \\
$\mathrm{C}$ & -2.114627 & -0.360922 & 1.488280 & $\mathrm{H}$ & -3.095173 & -0.129817 & 1.059981 \\
$\mathrm{C}$ & -0.601439 & 1.402699 & 2.447585 & $\mathrm{H}$ & -2.258684 & -0.525677 & 2.556975 \\
$\mathrm{C}$ & -0.194450 & 2.719609 & 2.432164 & $\mathrm{H}$ & 0.177921 & 3.179376 & 3.345268 \\
$\mathrm{C}$ & -0.159554 & 3.472567 & 1.226040 & $\mathrm{H}$ & 0.404531 & 5.336579 & 2.149418 \\
$\mathrm{C}$ & 0.222229 & 4.829346 & 1.205304 & $\mathrm{H}$ & 0.681536 & 6.551039 & 0.000000 \\
$\mathrm{C}$ & 0.389884 & 5.504104 & 0.000000 & $\mathrm{H}$ & 0.404531 & 5.336579 & -2.149418 \\
$\mathrm{C}$ & 0.222229 & 4.829346 & -1.205304 & $\mathrm{H}$ & -0.177921 & -3.179376 & 3.345268 \\
$\mathrm{C}$ & -0.159554 & 3.472567 & -1.226040 & $\mathrm{H}$ & 0.177921 & 3.179376 & -3.345268 \\
$\mathrm{C}$ & -0.417547 & 2.784025 & 0.000000 & $\mathrm{H}$ & 1.783998 & 1.310040 & -1.057753 \\
$\mathrm{C}$ & 0.956631 & -1.439675 & 0.000000 & $\mathrm{H}$ & 3.095173 & 0.129817 & -1.059981 \\
$\mathrm{C}$ & 0.194450 & -2.719609 & 2.432164 & $\mathrm{H}$ & 2.258684 & 0.525677 & -2.556975 \\
$\mathrm{C}$ & -0.956631 & 1.439675 & 0.000000 & $\mathrm{H}$ & -0.404531 & -5.336579 & 2.149418 \\
$\mathrm{C}$ & -0.194450 & 2.719609 & -2.432164 & $\mathrm{H}$ & -1.783998 & -1.310040 & -1.057753 \\
$\mathrm{C}$ & 1.163329 & -0.796317 & -1.272245 & $\mathrm{H}$ & -3.095173 & -0.129817 & -1.059981 \\
$\mathrm{C}$ & 0.417547 & -2.784025 & 0.000000 & $\mathrm{H}$ & -2.258684 & -0.525677 & -2.556975
\end{tabular}




\begin{tabular}{|c|c|c|c|c|c|c|c|}
\hline C & 0.159554 & -3.472567 & 1.226040 & $\mathrm{H}$ & -0.481242 & 1.085684 & -4.621206 \\
\hline C & -1.163329 & 0.796317 & -1.272245 & $\mathrm{H}$ & 0.481242 & -1.085684 & -4.621206 \\
\hline C & -0.601439 & 1.402699 & -2.447585 & $\mathrm{H}$ & -0.177921 & -3.179376 & -3.345268 \\
\hline C & 2.114627 & 0.360922 & -1.488280 & $\mathrm{H}$ & -0.404531 & -5.336579 & -2.149418 \\
\hline C & 0.601439 & -1.402699 & -2.447585 & $\mathrm{H}$ & -0.681536 & -6.551039 & 0.000000 \\
\hline C & 0.159554 & -3.472567 & -1.226040 & & & & \\
\hline C & -0.222229 & -4.829346 & 1.205304 & & & & \\
\hline C & -2.114627 & -0.360922 & -1.488280 & & & & \\
\hline C & -0.296972 & 0.606464 & -3.660099 & & & & \\
\hline C & 0.296972 & -0.606464 & -3.660099 & & & & \\
\hline C & 0.194450 & -2.719609 & -2.432164 & & & & \\
\hline C & -0.222229 & -4.829346 & -1.205304 & & & & \\
\hline C & -0.389884 & -5.504104 & 0.000000 & & & & \\
\hline
\end{tabular}

RB3LYP/6-311+G(2d) singlet of 10, $\mathrm{C}_{2 h}$ symmetry:

\begin{tabular}{|c|c|c|c|c|c|c|c|}
\hline & $\mathrm{x}$ & $y$ & z & & $\mathrm{x}$ & $y$ & z \\
\hline C & -0.295539 & 0.602894 & 3.646418 & $\mathrm{H}$ & -0.484352 & 1.076751 & 4.606275 \\
\hline C & 0.295539 & -0.602894 & 3.646418 & $\mathrm{H}$ & 0.484352 & -1.076751 & 4.606275 \\
\hline C & 0.601708 & -1.400297 & 2.438646 & $\mathrm{H}$ & 1.795596 & 1.298360 & 1.057329 \\
\hline C & 1.169854 & -0.801237 & 1.269002 & $\mathrm{H}$ & 3.096332 & 0.121357 & 1.058908 \\
\hline C & 2.119375 & 0.350478 & 1.485282 & $\mathrm{H}$ & 2.268103 & 0.516258 & 2.548734 \\
\hline C & -1.169854 & 0.801237 & 1.269002 & $\mathrm{H}$ & -1.795596 & -1.298360 & 1.057329 \\
\hline C & -2.119375 & -0.350478 & 1.485282 & $\mathrm{H}$ & -3.096332 & -0.121357 & 1.058908 \\
\hline C & -0.601708 & 1.400297 & 2.438646 & $\mathrm{H}$ & -2.268103 & -0.516258 & 2.548734 \\
\hline C & -0.193053 & 2.711169 & 2.423594 & $\mathrm{H}$ & 0.180886 & 3.167406 & 3.335070 \\
\hline C & -0.159059 & 3.463556 & 1.222421 & $\mathrm{H}$ & 771 & 5.322198 & 2.141732 \\
\hline C & 0.226345 & 4.814874 & 1.200917 & $\mathrm{H}$ & 207 & 6.529490 & 0.000000 \\
\hline C & 0.394741 & 5.486013 & 0.000000 & $\mathrm{H}$ & 0.4 & 5.322198 & -2.141732 \\
\hline C & 0.226345 & 4.814874 & -1.200917 & $\mathrm{H}$ & -0.1 & -3.167406 & 3.335070 \\
\hline C & -0.159059 & 3.463556 & -1.222421 & $\mathrm{H}$ & 0.1 & 3.167406 & -3.335070 \\
\hline C & -0.420320 & 2.779854 & 0.000000 & $\mathrm{H}$ & 1.7 & 1.298360 & -1.057329 \\
\hline C & 0.961719 & -1.441337 & 0.000000 & $\mathrm{H}$ & 332 & 0.121357 & -1.058908 \\
\hline C & 0.193053 & -2.711169 & 2.423594 & $\mathrm{H}$ & 2.268103 & 0.516258 & -2.548734 \\
\hline C & -0.961719 & 1.441337 & 0.000000 & $\mathrm{H}$ & -0.410771 & -5.322198 & 2.141732 \\
\hline C & -0.193053 & 2.711169 & -2.423594 & $\mathrm{H}$ & -1.7 & -1.298360 & -1.057329 \\
\hline C & 1.169854 & -0.801237 & -1.269002 & $\mathrm{H}$ & -3.0 & -0.121357 & -1.058908 \\
\hline C & 0.420320 & -2.779854 & 0.000000 & $\mathrm{H}$ & -2.26 & -0.516258 & -2.548734 \\
\hline C & 0.159059 & -3.463556 & 1.222421 & $\mathrm{H}$ & -0.48 & 1.076751 & -4.606275 \\
\hline C & -1.169854 & 0.801237 & -1.269002 & $\mathrm{H}$ & 0.484352 & -1.076751 & -4.606275 \\
\hline C & -0.601708 & 1.400297 & -2.438646 & $\mathrm{H}$ & -0.180886 & -3.167406 & -3.335070 \\
\hline C & 2.119375 & 0.350478 & -1.485282 & $\mathrm{H}$ & -0.410771 & -5.322198 & -2.141732 \\
\hline C & 0.601708 & -1.400297 & -2.438646 & $\mathrm{H}$ & -0.689207 & -6.529490 & 0.000000 \\
\hline C & 0.159059 & -3.463556 & -1.222421 & & & & \\
\hline C & -0.226345 & -4.814874 & 1.200917 & & & & \\
\hline C & -2.119375 & -0.350478 & -1.485282 & & & & \\
\hline C & -0.295539 & 0.602894 & -3.646418 & & & & \\
\hline C & 0.295539 & -0.602894 & -3.646418 & & & & \\
\hline C & 0.193053 & -2.711169 & -2.423594 & & & & \\
\hline C & -0.226345 & -4.814874 & -1.200917 & & & & \\
\hline C & -0.394741 & -5.486013 & 0.000000 & & & & \\
\hline
\end{tabular}


UB3LYP/6-31G* singlet of 10, $\mathrm{C}_{2 h}$ symmetry:

\begin{tabular}{|c|c|c|c|c|c|c|}
\hline & $\mathrm{x}$ & $y$ & $z$ & $x$ & $y$ & \\
\hline C & -0.308046 & 0.601533 & $3.630164 \mathrm{H}$ & -0.493703 & 1.069563 & 4.597116 \\
\hline C & 0.308046 & -0.601533 & $3.630164 \mathrm{H}$ & 0.493703 & -1.069563 & 4.597116 \\
\hline C & 0.633075 & -1.413328 & $2.430135 \mathrm{H}$ & 1.784350 & 1.274609 & 1.051001 \\
\hline C & 1.252903 & -0.851963 & $1.273457 \mathrm{H}$ & 3.146467 & 0.153548 & 1.055317 \\
\hline C & 2.157327 & 0.342676 & $1.483456 \mathrm{H}$ & 2.293200 & 0.517173 & 2.552005 \\
\hline C & -1.252903 & 0.851963 & $1.273457 \mathrm{H}$ & -1.784350 & -1.274609 & 1.051001 \\
\hline C & -2.157327 & -0.342676 & $1.483456 \mathrm{H}$ & -3.146467 & -0.153548 & 1.055317 \\
\hline C & -0.633075 & 1.413328 & $2.430135 \mathrm{H}$ & -2.293200 & -0.517173 & 2.552005 \\
\hline C & -0.199978 & 2.731399 & $2.422871 \mathrm{H}$ & 0.197557 & 3.172030 & 3.334700 \\
\hline C & -0.168071 & 3.491049 & 1.22 & 0.4 & 5.33 & 598 \\
\hline C & 0.257771 & 4.839226 & $1.205815 \mathrm{H}$ & 0.768397 & 704 & 0.000000 \\
\hline C & 0.443581 & 5.508566 & $0.000000 \mathrm{H}$ & 0.461470 & 5.338740 & -2.149598 \\
\hline C & 0.257771 & 4.839226 & $-1.205815 \mathrm{H}$ & -0.197557 & -3.172030 & 3.334700 \\
\hline C & -0.168071 & 3.491049 & -1.22 & 0.15 & 3.172030 & -3.334700 \\
\hline C & -0.462913 & 2.818184 & 0.00 & 1.784350 & 1.27 & -1.0 \\
\hline C & 1.042684 & -1.494473 & $0.000000 \mathrm{H}$ & 3.146467 & 0.153548 & -1.055317 \\
\hline C & 0.199978 & -2.731399 & $2.422871 \mathrm{H}$ & 2.293200 & 0.517173 & -2.552005 \\
\hline C & -1.042684 & 1.494473 & $0.000000 \mathrm{H}$ & -0.461470 & -5.338740 & 2.149598 \\
\hline C & -0.199978 & 2.731399 & $-2.422871 \mathrm{H}$ & -1.784350 & -1.274609 & -1.051001 \\
\hline C & 1.252903 & -0.851963 & $-1.273457 \mathrm{H}$ & -3.146467 & -0.153548 & -1.055317 \\
\hline C & 0.462913 & -2.818184 & $0.000000 \mathrm{H}$ & -2.293200 & -0.517173 & -2.552005 \\
\hline C & 0.168071 & -3.491049 & $1.227184 \mathrm{H}$ & -0.493703 & 1.069563 & -4.597116 \\
\hline C & -1.252903 & 0.851963 & $-1.273457 \mathrm{H}$ & 0.493703 & -1.069563 & -4.597116 \\
\hline C & -0.633075 & 1.413328 & $-2.430135 \mathrm{H}$ & -0.197557 & -3.172030 & -3.334700 \\
\hline C & 2.157327 & 0.342676 & -1.483 & -0.461470 & -5.338740 & -2.149598 \\
\hline C & 0.633075 & -1.413328 & $-2.430135 \mathrm{H}$ & -0.768397 & -6.545704 & 0.000000 \\
\hline C & 0.168071 & -3.491049 & -1.227184 & & & \\
\hline C & -0.257771 & -4.839226 & 1.205815 & & & \\
\hline C & -2.157327 & -0.342676 & -1.483456 & & & \\
\hline C & -0.308046 & 0.601533 & -3.630164 & & & \\
\hline C & 0.308046 & -0.601533 & -3.630164 & & & \\
\hline C & 0.199978 & -2.731399 & -2.422871 & & & \\
\hline C & -0.257771 & -4.839226 & -1.205815 & & & \\
\hline C & -0.443581 & -5.508566 & 0.000000 & & & \\
\hline
\end{tabular}

RB3LYP/6-31G* singlet of 11, $\mathrm{C}_{2 h}$ symmetry:

\begin{tabular}{|l|rrr|r|rrr|}
\hline & $\mathrm{x}$ & \multicolumn{2}{|c|}{$\mathrm{y}$} & $\mathrm{z}$ & $\mathrm{y}$ & $\mathrm{z}$ \\
\hline $\mathrm{C}$ & 0.019074 & 0.695055 & $3.762188 \mathrm{H}$ & 0.072892 & 1.231502 & 4.707458 \\
$\mathrm{C}$ & -0.019074 & -0.695055 & $3.762188 \mathrm{H}$ & -0.072892 & -1.231502 & 4.707458 \\
$\mathrm{C}$ & 0.059194 & -1.446790 & 2.560556 & $\mathrm{H}$ & 2.302336 & 0.534845 & 0.946597 \\
$\mathrm{C}$ & 0.426745 & -0.649274 & 1.307885 & $\mathrm{H}$ & 2.557965 & -1.201328 & 1.212071 \\
$\mathrm{C}$ & 1.965770 & -0.333141 & 1.511005 & $\mathrm{H}$ & 2.166832 & -0.135825 & 2.564539 \\
$\mathrm{C}$ & -0.426745 & 0.649274 & 1.307885 & $\mathrm{H}$ & -2.302336 & -0.534845 & 0.946597 \\
$\mathrm{C}$ & -1.965770 & 0.333141 & 1.511005 & $\mathrm{H}$ & -2.557965 & 1.201328 & 1.212071 \\
$\mathrm{C}$ & -0.059194 & 1.446790 & $2.560556 \mathrm{H}$ & -2.166832 & 0.135825 & 2.564539
\end{tabular}




\begin{tabular}{|c|c|c|c|c|c|c|}
\hline C & 0.036471 & 2.822453 & $2.493449 \mathrm{H}$ & 0.189937 & 3.392661 & 3.408857 \\
\hline C & 0.006334 & 3.525130 & $1.266139 \mathrm{H}$ & 0.195930 & 5.470898 & 2.150050 \\
\hline C & 0.126994 & 4.918477 & $1.215862 \mathrm{H}$ & 0.265342 & 6.687430 & 0.000000 \\
\hline C & 0.172422 & 5.604881 & $0.000000 \mathrm{H}$ & 0.195930 & 5.470898 & -2.150050 \\
\hline C & 0.126994 & 4.918477 & $-1.215862 \mathrm{H}$ & -0.189937 & -3.392661 & 3.408857 \\
\hline C & 0.006334 & 3.525130 & $-1.266139 \mathrm{H}$ & 0.189937 & 3.392661 & -3.408857 \\
\hline C & -0.100396 & 2.773783 & $0.000000 \mathrm{H}$ & 2.302336 & 0.534845 & -0.946597 \\
\hline C & 0.251479 & -1.420901 & $0.000000 \mathrm{H}$ & 2.557965 & -1.201328 & -1.212071 \\
\hline C & -0.036471 & -2.822453 & $2.493449 \mathrm{H}$ & 2.166832 & -0.135825 & -2.564539 \\
\hline C & -0.251479 & 1.420901 & $0.000000 \mathrm{H}$ & -0.195930 & -5.470898 & 2.150050 \\
\hline C & 0.036471 & 2.822453 & $-2.493449 \mathrm{H}$ & -2.302336 & -0.534845 & -0.946597 \\
\hline C & 0.426745 & -0.649274 & $-1.307885 \mathrm{H}$ & -2.557965 & 1.201328 & -1.212071 \\
\hline C & 0.100396 & -2.773783 & $0.000000 \mathrm{H}$ & -2.166832 & 0.135825 & -2.564539 \\
\hline C & -0.006334 & -3.525130 & $1.266139 \mathrm{H}$ & 0.072892 & 1.231502 & -4.707458 \\
\hline c & -0.426745 & 0.649274 & $-1.307885 \mid \mathrm{H}$ & -0.072892 & -1.231502 & -4.707458 \\
\hline C & -0.059194 & 1.446790 & $-2.560556 \mathrm{H}$ & -0.189937 & -3.392661 & -3.408857 \\
\hline C & 1.965770 & -0.333141 & $-1.511005 \mathrm{H}$ & -0.195930 & -5.470898 & -2.150050 \\
\hline C & 0.059194 & -1.446790 & $-2.560556 \mathrm{H}$ & -0.265342 & -6.687430 & 0.000000 \\
\hline C & -0.006334 & -3.525130 & -1.266139 & & & \\
\hline C & -0.126994 & -4.918477 & 1.215862 & & & \\
\hline C & -1.965770 & 0.333141 & -1.511005 & & & \\
\hline C & 0.019074 & 0.695055 & -3.762188 & & & \\
\hline C & -0.019074 & -0.695055 & -3.762188 & & & \\
\hline C & -0.036471 & -2.822453 & -2.493449 & & & \\
\hline C & -0.126994 & -4.918477 & -1.215862 & & & \\
\hline C & -0.172422 & -5.604881 & 0.000000 & & & \\
\hline
\end{tabular}

UB3LYP/6-31G* singlet of 11, $\mathrm{C}_{2 h}$ symmetry:

\begin{tabular}{|c|c|c|c|c|c|c|}
\hline & $x$ & 1 & & $x$ & & \\
\hline C & -0.025084 & 0.683698 & $3.772346 \mathrm{H}$ & -0.014021 & 1.227487 & 4.7149 \\
\hline C & 084 & 698 & $\mathrm{H}$ & & -1.22 & \\
\hline C & 0.122018 & -1.447975 & 2.547 & 049 & 0.6 & 100 \\
\hline C & 0.469647 & -0.639694 & $1.302284 \mathrm{H}$ & 2.634795 & -1.021533 & 1.181818 \\
\hline C & 1.972479 & -0.216023 & 1.50 & 2.1 & -0.0 & 54 \\
\hline C & & & & -2.2 & -0.6 & \\
\hline C & -1.9 & 0.21 & 1.5 & -2.6 & & 318 \\
\hline C & -0.12 & 1.4 & 2.5 & -2.1 & 0.0 & 549 \\
\hline C & 75 & 2.8 & 2.4 & 0. & 3.3 & 131 \\
\hline C & -0.0 & 3.5 & 1.24 & 0.1 & 5.4 & 212 \\
\hline C & & & 1.2 & & & 000 \\
\hline C & & & 0.0 & & & 212 \\
\hline C & 83 & 4.9 & -1.2 & -0.1 & -3.3 & 2131 \\
\hline C & -0 . & & -1.2 & & 3.3 & 131 \\
\hline C & -0.1 & 2.7 & 0.0 & 49 & 0.6 & -0. \\
\hline C & & & & & & \\
\hline C & -0.006475 & -2.80 & $2.4 \varepsilon$ & & -0.0 & 4549 \\
\hline C & -0.309586 & 1.409557 & $0.000000 \mathrm{H}$ & -0.197335 & -5.460179 & 2.151212 \\
\hline
\end{tabular}




\begin{tabular}{l|rrr|rrr|}
$\mathrm{C}$ & 0.006475 & 2.802723 & -2.487067 \\
$\mathrm{C}$ & 0.469647 & -0.639694 & $-1.302284 \mathrm{H}$ & -2.226049 & -0.692646 & -0.963160 \\
$\mathrm{C}$ & 0.121195 & -2.788338 & $0.000000 \mathrm{H}$ & -2.171017 & 0.028830 & -2.564549 \\
$\mathrm{C}$ & 0.013508 & -3.526348 & $1.245273 \mathrm{H}$ & -0.014021 & 1.227487 & -4.714939 \\
$\mathrm{C}$ & -0.469647 & 0.639694 & $-1.302284 \mathrm{H}$ & 0.014021 & -1.227487 & -4.714939 \\
$\mathrm{C}$ & -0.122018 & 1.447975 & $-2.547187 \mathrm{H}$ & -0.177596 & -3.368690 & -3.402131 \\
$\mathrm{C}$ & 1.972479 & -0.216023 & $-1.508713 \mathrm{H}$ & -0.197335 & -5.460179 & -2.151212 \\
$\mathrm{C}$ & 0.122018 & -1.447975 & $-2.547187 \mathrm{H}$ & -0.281547 & -6.694519 & 0.000000 \\
$\mathrm{C}$ & 0.013508 & -3.526348 & -1.245273 \\
$\mathrm{C}$ & -0.123283 & -4.917668 & 1.211476 \\
$\mathrm{C}$ & -1.972479 & 0.216023 & -1.508713 \\
$\mathrm{C}$ & -0.025084 & 0.683698 & -3.772346 \\
$\mathrm{C}$ & 0.025084 & -0.683698 & -3.772346 \\
$\mathrm{C}$ & -0.006475 & -2.802723 & -2.487067 \\
$\mathrm{C}$ & -0.123283 & -4.917668 & -1.211476 \\
$\mathrm{C}$ & -0.177897 & -5.613246 & 0.000000 \\
$\mathrm{l}$
\end{tabular}

$\pi$-bonded structure of $\mathbf{1 3}$ by RB3LYP/6-31G*, singlet, $\mathrm{C}_{2 h}$ symmetry:

\begin{tabular}{|l|rrr|r|rrr|}
\hline & $x$ & \multicolumn{3}{|c}{$\mathrm{y}$} & $\mathrm{z}$ & $\mathrm{x}$ & $\mathrm{y}$ \\
\hline $\mathrm{C}$ & -0.588092 & 0.524027 & $3.754444 \mathrm{H}$ & 1.760207 & 1.414897 & 1.038896 \\
$\mathrm{C}$ & 0.588092 & -0.524027 & $3.754444 \mathrm{H}$ & 3.137788 & 0.313237 & 0.985885 \\
$\mathrm{C}$ & 0.738365 & -1.318430 & $2.466423 \mathrm{H}$ & 2.364031 & 0.681259 & 2.505440 \\
$\mathrm{C}$ & 1.264731 & -0.714773 & $1.278265 \mathrm{H}$ & -1.760207 & -1.414897 & 1.038896 \\
$\mathrm{C}$ & 2.165605 & 0.488606 & $1.453262 \mathrm{H}$ & -3.137788 & -0.313237 & 0.985885 \\
$\mathrm{C}$ & -1.264731 & 0.714773 & $1.278265 \mathrm{H}$ & -2.364031 & -0.681259 & 2.505440 \\
$\mathrm{C}$ & -2.165605 & -0.488606 & $1.453262 \mathrm{H}$ & 0.082517 & 3.082306 & 3.352233 \\
$\mathrm{C}$ & -0.738365 & 1.318430 & $2.466423 \mathrm{H}$ & 0.432556 & 5.205375 & 2.149812 \\
$\mathrm{C}$ & -0.270924 & 2.615357 & $2.435090 \mathrm{H}$ & 0.755933 & 6.406670 & 0.000000 \\
$\mathrm{C}$ & -0.195245 & 3.360281 & $1.227476 \mathrm{H}$ & 0.432556 & 5.205375 & -2.149812 \\
$\mathrm{C}$ & 0.235156 & 4.703498 & $1.205811 \mathrm{H}$ & -0.082517 & -3.082306 & 3.352233 \\
$\mathrm{C}$ & 0.427832 & 5.370388 & $0.000000 \mathrm{H}$ & 0.082517 & 3.082306 & -3.352233 \\
$\mathrm{C}$ & 0.235156 & 4.703498 & $-1.205811 \mathrm{H}$ & 1.760207 & 1.414897 & -1.038896 \\
$\mathrm{C}$ & -0.195245 & 3.360281 & $-1.227476 \mathrm{H}$ & 3.137788 & 0.313237 & -0.985885 \\
$\mathrm{C}$ & -0.478678 & 2.682481 & $0.000000 \mathrm{H}$ & 2.364031 & 0.681259 & -2.505440 \\
$\mathrm{C}$ & 1.045730 & -1.353828 & $0.000000 \mathrm{H}$ & -0.432556 & -5.205375 & 2.149812 \\
$\mathrm{C}$ & 0.270924 & -2.615357 & $2.435090 \mathrm{H}$ & -1.760207 & -1.414897 & -1.038896 \\
$\mathrm{C}$ & -1.045730 & 1.353828 & $0.000000 \mathrm{H}$ & -3.137788 & -0.313237 & -0.985885 \\
$\mathrm{C}$ & -0.270924 & 2.615357 & $-2.435090 \mathrm{H}$ & -2.364031 & -0.681259 & -2.505440 \\
$\mathrm{C}$ & 1.264731 & -0.714773 & $-1.278265 \mathrm{H}$ & -0.082517 & -3.082306 & -3.352233 \\
$\mathrm{C}$ & 0.478678 & -2.682481 & $0.000000 \mathrm{H}$ & -0.432556 & -5.205375 & -2.149812 \\
$\mathrm{C}$ & 0.195245 & -3.360281 & $1.227476 \mathrm{H}$ & -0.755933 & -6.406670 & 0.000000 \\
$\mathrm{C}$ & -1.264731 & 0.714773 & $-1.278265 \mathrm{H}$ & -0.404098 & 1.221321 & 4.579578 \\
$\mathrm{C}$ & -0.738365 & 1.318430 & $-2.466423 \mathrm{H}$ & -1.510872 & -0.003525 & 4.012440 \\
$\mathrm{C}$ & 2.165605 & 0.488606 & $-1.453262 \mathrm{H}$ & 0.404098 & -1.221321 & 4.579578 \\
$\mathrm{C}$ & 0.738365 & -1.318430 & $-2.466423 \mathrm{H}$ & 1.510872 & 0.003525 & 4.012440 \\
$\mathrm{C}$ & 0.195245 & -3.360281 & $-1.227476 \mathrm{H}$ & -1.510872 & -0.003525 & -4.012440 \\
$\mathrm{C}$ & -0.235156 & -4.703498 & $1.205811 \mathrm{H}$ & -0.404098 & 1.221321 & -4.579578 \\
& & & & & &
\end{tabular}




\begin{tabular}{|l|rrr|rrr|}
$C$ & -2.165605 & -0.488606 & -1.453262 \\
$C$ & -0.588092 & 0.524027 & -3.754444 \\
$C$ & $-H$ & 0.404098 & -1.221321 & -4.579578 \\
$C$ & 0.588092 & -0.524027 & -3.754444 \\
$C$ & 0.270924 & -2.615357 & -2.435090 \\
$C$ & -0.235156 & -4.703498 & -1.205811 \\
$C$ & -0.427832 & -5.370388 & 0.000000 \\
\hline
\end{tabular}

$\pi$-bonded structure of 13 by UB3LYP/6-31G*, singlet, $\mathrm{C}_{2 h}$ symmetry:

\begin{tabular}{|c|c|c|c|c|c|c|}
\hline & $x$ & $y$ & & $x$ & $y$ & \\
\hline C & -0.653726 & 0.438407 & $3.716100 \mathrm{H}$ & 1.927640 & 1.418836 & 1.058252 \\
\hline C & 0.653726 & -0.438407 & $3.716100 \mathrm{H}$ & 3.322352 & 0.340215 & 0.964948 \\
\hline C & 0.855189 & -1.257995 & $2.448915 \mathrm{H}$ & 2.577153 & 0.671527 & 2.503455 \\
\hline C & 1.464016 & -0.717385 & $1.281457 \mathrm{H}$ & -1.927640 & -1.418836 & 1.058252 \\
\hline C & 2.357645 & 0.493934 & $1.451807 \mathrm{H}$ & -3.322352 & -0.340215 & 0.964948 \\
\hline C & -1.464016 & 0.717385 & 1.28 & -2.577153 & -0.671527 & 3455 \\
\hline C & -2.357645 & -0.493934 & $1.451807 \mathrm{H}$ & 0.066686 & 2.976991 & 3.340528 \\
\hline C & -0.855189 & 1.257995 & $2.448915 \mathrm{H}$ & 0.510248 & 5.094018 & 2.149804 \\
\hline C & -0.327929 & 2.542023 & $2.424313 \mathrm{H}$ & 0.899461 & 6.277868 & 0.000000 \\
\hline C & -0.235440 & 3.28 & 1.2 & 0.510248 & 5.0 & 804 \\
\hline C & 0.277966 & 4.608202 & $1.2 C$ & -0.066686 & -2.97 & 528 \\
\hline C & 0.508406 & 5.263699 & $0.000000 \mathrm{H}$ & 1.927640 & 1.41 & -1.058252 \\
\hline C & 0.277966 & 4.608202 & -1.20 & 3.322352 & 0.34 & -0.964948 \\
\hline C & -0.235440 & 3.288663 & -1.22 & 2.577153 & 0.67 & -2.5 \\
\hline C & -0.574066 & 2.63 & 0.0 & 0.066686 & 2.9 & 528 \\
\hline C & 1.22 & -1.346513 & 0.0 & -0.510248 & -5.0 & 9804 \\
\hline C & 0.327929 & -2.542023 & 2.4 & -1.927640 & -1.418836 & -1.058252 \\
\hline C & -1.221144 & 1.346513 & 0.00 & -3.322352 & -0.3 & -0.964948 \\
\hline C & -0.327929 & 2.542023 & $-2.4 z$ & -2.577153 & -0.67 & -2.503455 \\
\hline C & 1.464016 & -0.717385 & $-1.2 \varepsilon$ & -0.066686 & -2.97 & -3.340528 \\
\hline C & 0.57 & -2.6 & 0.0 & 248 & -5.0 & -2.149804 \\
\hline C & 0.235440 & -3.288663 & 1.2 & 461 & -6.27 & 0.000000 \\
\hline C & -1.464016 & 0.717385 & -1.28 & -0.585917 & 1.116490 & 4.575476 \\
\hline C & -0.855189 & 1.257995 & $-2.448915 \mathrm{H}$ & -1.500380 & -0.220990 & 3.919548 \\
\hline C & 645 & 0.493934 & -1.45 & 0.585917 & -1.116490 & 4.575476 \\
\hline C & 0.855189 & -1.25 & -2.4 & 380 & 0.2 & 3.919548 \\
\hline C & 0.235440 & -3.288663 & -1.22 & -1.500380 & -0.220990 & -3.919548 \\
\hline C & -0.277966 & -4.608202 & $1.205388 \mathrm{H}$ & -0.585917 & 1.116490 & -4.575476 \\
\hline C & -2.357645 & -0.493934 & -1.45 & 1.500380 & 0.220990 & -3.919548 \\
\hline C & -0.653726 & 0.438407 & $-3.716100 \mathrm{H}$ & 0.585917 & -1.116490 & -4.575476 \\
\hline C & 0.653726 & -0.438407 & -3.716100 & & & \\
\hline C & 0.327929 & -2.542023 & -2.42 & & & \\
\hline C & -0.277966 & -4.608202 & -1.20 & & & \\
\hline C & -0.508406 & -5.263699 & 0.000000 & & & \\
\hline
\end{tabular}

$\sigma$-bonded structure of 13 by R(U)B3LYP/6-31G*, singlet, $\mathrm{C}_{2}$ symmetry:

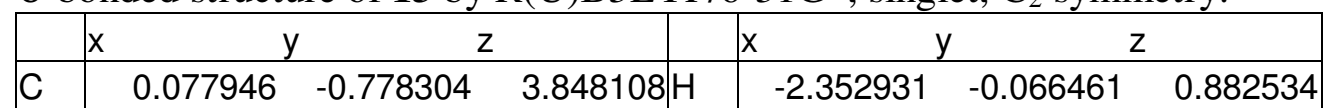




\begin{tabular}{|c|c|c|c|c|c|c|}
\hline C & -0.077946 & 0.778304 & $3.848108 \mathrm{H}$ & -2.349702 & 1.650183 & \\
\hline 10 & 0.268540 & 1.416490 & $2.518928 \mathrm{H}$ & -2.107782 & 0.394719 & 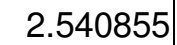 \\
\hline C & -0.350048 & 0.792588 & $1.278414 \mathrm{H}$ & 2.352931 & 0.066461 & 0.882 \\
\hline C & -1.878913 & 0.678388 & $1.516168 \mathrm{H}$ & 2.349702 & -1.650183 & 1.33 \\
\hline & 0.350048 & -0.792588 & $1.278414 \mathrm{H}$ & 2.107782 & -0.394719 & 2. \\
\hline & 1.878913 & -0.678388 & $1.516168 \mathrm{H}$ & -1.607806 & -2.812940 & 3.35 \\
\hline & -0.268540 & -1.416490 & $2.518928 \mathrm{H}$ & -3.019829 & -4.321213 & \\
\hline & -1.166280 & -2.416101 & $2.445078 \mathrm{H}$ & -3.899254 & -5.198012 & -0.0557 \\
\hline & -1.642188 & -2.959516 & $1.190429 \mathrm{H}$ & -3.024764 & -4.308427 & -2.2009 \\
\hline & -2.626531 & -3.939663 & $1.159558 \mathrm{H}$ & 1.607806 & 2.812940 & 3.358 \\
\hline 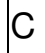 & -3.125628 & -4.434841 & $-0.059617 \mathrm{H}$ & -1.664504 & -2.623369 & -3.403 \\
\hline & -2.637451 & -3.944623 & $-1.252143 \mathrm{H}$ & -2.201307 & -0.313709 & -1.16 \\
\hline & -1.619123 & -2.957597 & $-1.267347 \mathrm{H}$ & -2.722855 & 1.324949 & -0.7662 \\
\hline C & -1.095328 & -2.452365 & $-0.034230 \mathrm{H}$ & -2.408084 & 0.889748 & -2.423 \\
\hline 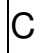 & 0.000000 & 1.524567 & $-0.027941 \mathrm{H}$ & 3.019829 & 4.321213 & 2.09902 \\
\hline C & 1.166280 & 2.416101 & $2.445078 \mathrm{H}$ & 2.201307 & 0.313 & -1.165976 \\
\hline j & 0.000000 & -1.524567 & $-0.027941 \mathrm{H}$ & 2.722855 & -1.324949 & -0.76 \\
\hline C & -1.149385 & -2.384273 & $-2.475030 \mathrm{H}$ & 2.408084 & -0.889748 & -2.423 \\
\hline C & -0.627628 & 1.241772 & $-1.260643 \mathrm{H}$ & 1.664504 & 2.623 & -3.4036 \\
\hline C & 1.095328 & 2.452365 & $-0.034230 \mathrm{H}$ & 3.024764 & 4.308427 & -2.200924 \\
\hline C & 1.642188 & 2.959516 & $1.190429 \mathrm{H}$ & 3.899254 & 5.198012 & -0.055719 \\
\hline C & 0.627628 & -1.241772 & $-1.260643 \mathrm{H}$ & -0.558299 & -1.205421 & 4.63 \\
\hline C & -0.076525 & -1.522876 & $-2.486993 \mathrm{H}$ & 1.108753 & -1.021437 & 4.133 \\
\hline C & -2.056282 & 0.742266 & $-1.402775 \mathrm{H}$ & 0.558299 & 1.205421 & 4.63 \\
\hline C & 0.076525 & 1.522876 & $-2.486993 \mathrm{H}$ & -1.108753 & 1.021437 & 4.133304 \\
\hline C & 1.619123 & 2.957597 & $-1.267347 \mathrm{H}$ & 1.323650 & -0.741275 & -3.9636 \\
\hline C & 2.626531 & 3.939663 & $1.159558 \mathrm{H}$ & -0.222102 & -1.246740 & -4.609970 \\
\hline C & 2.056282 & -0.742266 & $-1.402775 \mathrm{H}$ & -1.323650 & 0.741275 & -3.963649 \\
\hline C & 0.250103 & -0.748600 & $-3.755101 \mathrm{H}$ & 0.222102 & 1.246740 & -4.609970 \\
\hline C & -0.250103 & 0.748600 & -3.755101 & & & \\
\hline & 1.149385 & 2.384273 & -2.475030 & & & \\
\hline C & 2.637451 & 3.944623 & -1.252143 & & & \\
\hline & 3.125628 & 4.434841 & -0.059617 & & & \\
\hline
\end{tabular}

Neutral 14 singlet by R(U)B3LYP/6-31G*, $\mathrm{C}_{2 h}$ symmetry:

\begin{tabular}{|l|rrr|r|rrr|}
\hline & $x$ & $y$ & & \multicolumn{3}{c|}{$\mathrm{y}$} & $\mathrm{z}$ \\
\hline $\mathrm{C}$ & 0.635340 & 0.233056 & $3.593112 \mathrm{H}$ & 4.407974 & -0.881185 & 1.208283 \\
$\mathrm{C}$ & -0.635340 & -0.233056 & $3.593112 \mathrm{H}$ & 4.407974 & -0.881185 & -1.208283 \\
$\mathrm{C}$ & -1.494998 & -0.452706 & 2.399886 & $\mathrm{H}$ & 1.115740 & 0.354429 & 4.565233 \\
$\mathrm{C}$ & -1.082775 & -1.182745 & $1.278288 \mathrm{H}$ & -1.115740 & -0.354429 & 4.565233 \\
$\mathrm{C}$ & 0.012365 & -2.211515 & $1.473565 \mathrm{H}$ & 0.970469 & -1.937567 & 1.028479 \\
$\mathrm{C}$ & 1.082775 & 1.182745 & 1.278288 & $\mathrm{H}$ & -0.288332 & -3.174514 & 1.050896 \\
$\mathrm{C}$ & -0.012365 & 2.211515 & $1.473565 \mathrm{H}$ & 0.185493 & -2.363481 & 2.540398 \\
$\mathrm{C}$ & 1.494998 & 0.452706 & $2.399886 \mathrm{H}$ & -0.970469 & 1.937567 & 1.028479 \\
$\mathrm{C}$ & 2.743808 & -0.226869 & 2.386624 & $\mathrm{H}$ & 0.288332 & 3.174514 & 1.050896 \\
$\mathrm{C}$ & 3.462129 & -0.345321 & $1.226243 \mathrm{H}$ & -0.185493 & 2.363481 & 2.540398 \\
$\mathrm{C}$ & 3.462129 & -0.345321 & $-1.226243 \mathrm{H}$ & 3.099597 & -0.690449 & 3.303788
\end{tabular}




\begin{tabular}{l|rrr|rrr|}
$\mathrm{C}$ & 2.901614 & 0.099040 & $0.000000 \mathrm{H}$ & -3.099597 & 0.690449 & 3.303788 \\
$\mathrm{C}$ & -1.704518 & -0.901408 & $0.000000 \mathrm{H}$ & 3.099597 & -0.690449 & -3.303788 \\
$\mathrm{C}$ & -2.743808 & 0.226869 & $2.386624 \mathrm{H}$ & -4.407974 & 0.881185 & 1.208283 \\
$\mathrm{C}$ & 1.704518 & 0.901408 & $0.000000 \mathrm{H}$ & 0.970469 & -1.937567 & -1.028479 \\
$\mathrm{C}$ & 2.743808 & -0.226869 & $-2.386624 \mathrm{H}$ & -0.288332 & -3.174514 & -1.050896 \\
$\mathrm{C}$ & -1.082775 & -1.182745 & $-1.278288 \mathrm{H}$ & 0.185493 & -2.363481 & -2.540398 \\
$\mathrm{C}$ & -2.901614 & -0.099040 & $0.000000 \mathrm{H}$ & -4.407974 & 0.881185 & -1.208283 \\
$\mathrm{C}$ & -3.462129 & 0.345321 & $1.226243 \mathrm{H}$ & -0.970469 & 1.937567 & -1.028479 \\
$\mathrm{C}$ & 1.082775 & 1.182745 & $-1.278288 \mathrm{H}$ & 0.288332 & 3.174514 & -1.050896 \\
$\mathrm{C}$ & 1.494998 & 0.452706 & $-2.399886 \mathrm{H}$ & -0.185493 & 2.363481 & -2.540398 \\
$\mathrm{C}$ & 0.012365 & -2.211515 & $-1.473565 \mathrm{H}$ & 1.115740 & 0.354429 & -4.565233 \\
$\mathrm{C}$ & -1.494998 & -0.452706 & $-2.399886 \mathrm{H}$ & -1.115740 & -0.354429 & -4.565233 \\
$\mathrm{C}$ & -3.462129 & 0.345321 & $-1.226243 \mathrm{H}$ & -3.099597 & 0.690449 & -3.303788 \\
$\mathrm{C}$ & -0.012365 & 2.211515 & -1.473565 \\
$\mathrm{C}$ & 0.635340 & 0.233056 & -3.593112 \\
$\mathrm{C}$ & -0.635340 & -0.233056 & -3.593112 \\
$\mathrm{C}$ & -2.743808 & 0.226869 & -2.386624 \\
\hline
\end{tabular}

$\pi$-bonded 14 singlet dication by R(U)B3LYP/6-31G*, $\mathrm{C}_{2 h}$ symmetry:

\begin{tabular}{|c|c|c|c|c|c|c|}
\hline & $x$ & 1 & & $x$ & & \\
\hline C & 0.651298 & 0.193509 & $3.644679 \mathrm{H}$ & 4.414923 & -0.729494 & 1.201689 \\
\hline C & -0.651298 & -0.193509 & $3.644679 \mathrm{H}$ & 4.414923 & -0.729494 & -1.201689 \\
\hline C & -1.449613 & -0.396414 & $2.436452 \mathrm{H}$ & 1.168816 & 0.267363 & 4.599469 \\
\hline C & -0.945230 & -1.088858 & $1.271149 \mathrm{H}$ & -1.168816 & -0.267363 & 4.599469 \\
\hline C & 0.045502 & -2.204307 & $1.496584 \mathrm{H}$ & 1.010903 & -2.057793 & 1.004434 \\
\hline C & 0.945230 & 1.088858 & 1.27 & -0.371226 & -3.140194 & 1.108625 \\
\hline C & -0.045502 & 2.204307 & $584 \mathrm{H}$ & 0.229182 & -2.347001 & 2.560604 \\
\hline C & 1.449613 & 0.396414 & 2.43 & -1.010903 & 2.057793 & 1.004434 \\
\hline C & 2.743043 & -0.150150 & $2.415615 \mathrm{H}$ & 0.371226 & 3.140194 & 1.108625 \\
\hline C & 3.428634 & -0.274392 & 1.21 & -0.2 & 2.3 & 2.560604 \\
\hline C & 3.428634 & -0.274392 & -1.2 & 3.1 & -0.5 & 2347 \\
\hline C & 2.810936 & 0.080769 & 0.0 & -3.17 & 0.5 & 3.332347 \\
\hline C & -1.559344 & -0.799780 & $0.000000 \mathrm{H}$ & 520 & -0.543290 & -3.332347 \\
\hline C & -2.743043 & 0.150150 & 2.41 & -4.41 & 0.729494 & 1.201689 \\
\hline C & 1.559344 & 0.799780 & 0.0 & 903 & -2.057793 & -1.004434 \\
\hline C & 2.743043 & -0.15 & -2.4 & -0.3 & -3.1 & -1.1 \\
\hline C & -0.945230 & -1.088858 & -1.27 & 0.2 & -2.347001 & -2.560604 \\
\hline C & -2.810936 & -0.080769 & 0.00 & -4.4 & 0.729494 & -1.201689 \\
\hline C & -3.428634 & 0.274392 & $1.219355 \mathrm{H}$ & -1.010903 & 2.057793 & -1.004434 \\
\hline C & 230 & 858 & -1.2 & 226 & 3.1 & -1.108625 \\
\hline C & 1.44 & 0.3 & -2.4 & -0.2 & 001 & 604 \\
\hline C & 0.045502 & -2.204307 & $-1.496584 \mathrm{H}$ & 1.168816 & 0.267363 & -4.599469 \\
\hline C & -1.449613 & -0.396414 & $-2.436452 \mathrm{H}$ & -1.168816 & -0.267363 & -4.599469 \\
\hline C & -3.428634 & 0.274392 & $-1.219355 \mathrm{H}$ & -3.172520 & 0.543290 & -3.332347 \\
\hline C & -0.04 & 2.2 & -1.4 & & & \\
\hline C & 298 & 0.15 & -3.6 & & & \\
\hline C & -0.651298 & -0.193509 & -3.644679 & & & \\
\hline C & -2.743043 & 0.150150 & -2.415615 & & & \\
\hline
\end{tabular}


$\pi$-bonded 14 singlet dianion by R(U)B3LYP/6-31G*, $\mathrm{C}_{2 h}$ symmetry:

\begin{tabular}{|c|c|c|c|c|c|c|}
\hline & $\mathrm{x}$ & $z$ & 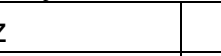 & $x$ & & \\
\hline C & 0.678337 & 0.141567 & $3.617507 \mathrm{H}$ & 4.513758 & -0.744862 & 1.216976 \\
\hline C & -0.678337 & -0.141567 & $3.617507 \mathrm{H}$ & 4.513758 & -0.744862 & -1.216976 \\
\hline C & -1.534179 & -0.395690 & $2.459892 \mathrm{H}$ & 1.177241 & 0.138418 & 4.594900 \\
\hline C & -1.047899 & -1.055801 & $1.281976 \mathrm{H}$ & -1.177241 & -0.138418 & 4.594900 \\
\hline C & 0.013524 & -2.120396 & $1.495366 \mathrm{H}$ & 1.007645 & -1.871349 & 1.113876 \\
\hline C & 1.047899 & 1.055801 & $1.281976 \mathrm{H}$ & -0.287763 & -3.071801 & 6846 \\
\hline C & -0.013524 & 2.120396 & $1.495366 \mathrm{H}$ & 0.124899 & -2.302280 & 2.568757 \\
\hline C & 1.534179 & 0.395690 & $2.459892 \mathrm{H}$ & -1.007645 & 1.871349 & 1.113876 \\
\hline C & 2.825039 & -0.166242 & $2.429405 \mathrm{H}$ & 0.287763 & 3.071801 & 1.036846 \\
\hline C & 92 & -0.27 & $86 \mathrm{H}$ & -0.1 & 2.3 & 757 \\
\hline C & 3.529892 & -0.271807 & $586 \mathrm{H}$ & 746 & -0.606601 & 766 \\
\hline C & 2.916939 & 0.065043 & $0.000000 \mathrm{H}$ & -3.229746 & 0.606601 & 3.344766 \\
\hline C & -1.636893 & -0.767355 & $000 \mathrm{H}$ & 3.2 & -0.60 & -3.344766 \\
\hline C & -2.825039 & 242 & $05 \mathrm{H}$ & -4.5 & 0.7 & 9976 \\
\hline C & 1.63 & 355 & $00 \mathrm{H}$ & 645 & -1.8 & -1.1 \\
\hline C & 2.825039 & -0.16 & $05 \mathrm{H}$ & -0.2 & -3.0 & -1.0 \\
\hline C & -1.047899 & -1.055801 & $76 \mathrm{H}$ & 399 & -2.3 & -2.568757 \\
\hline C & -2.916939 & -0.065043 & $0.000000 \mathrm{H}$ & -4.513758 & 0.74 & -1.216976 \\
\hline C & -3.529892 & 807 & $586 \mathrm{H}$ & -1.007645 & 1.871349 & 3876 \\
\hline C & 1.047899 & 1.055801 & $-1.281976 \mathrm{H}$ & 0.287763 & 3.071801 & -1.036846 \\
\hline C & 1.534179 & 90 & $892 \mathrm{H}$ & -0.1 & 280 & -2.568757 \\
\hline C & 0.013524 & -2.120396 & $-1.495366 \mathrm{H}$ & 1.177241 & 0.138418 & -4.594900 \\
\hline C & -1.534179 & -0.395690 & $-2.459892 \mathrm{H}$ & -1.177241 & -0.138418 & -4.594900 \\
\hline C & -3.529892 & 0.271807 & $-1.237586 \mathrm{H}$ & -3.229746 & 0.606601 & -3.344766 \\
\hline C & -0.013524 & 2.120396 & -1.4 & & & \\
\hline C & 0.678337 & 567 & -3.61 & & & \\
\hline C & -0.678337 & -0.141567 & -3.617507 & & & \\
\hline C & -2.825039 & 0.166242 & -2.429405 & & & \\
\hline
\end{tabular}

$\pi$-bonded 14 singlet dianion by $\mathrm{R}(\mathrm{U}) \mathrm{B} 3 \mathrm{LYP} / 6-31 \mathrm{G}^{*}, \mathrm{C}_{2}$ symmetry:

\begin{tabular}{|c|c|c|c|c|c|c|}
\hline & $\mathrm{x}$ & $z$ & $z$ & $\mathrm{x}$ & z & \\
\hline C & 0.693388 & -0.096790 & $-3.595300 \mathrm{H}$ & 4.540561 & 0.739420 & -1.184980 \\
\hline C & -0.693388 & 0.096790 & $-3.595300 \mathrm{H}$ & 4.486440 & 0.779362 & 3063 \\
\hline C & -1.545249 & 0.347147 & $-2.453117 \mathrm{H}$ & 1.200190 & -0.014683 & -4.566326 \\
\hline C & -1.0 & 1.05 & -1.28 & -1.2 & 0.0 & -4 \\
\hline C & -0.06 & 2.1 & -1.5 & 0.9 & 34 & -1 \\
\hline C & 1.074043 & -1.05 & -1.28 & -0.2 & 35 & $-0 . \varepsilon$ \\
\hline C & 0.069654 & -2.15 & -1.55 & -0.2( & 2.5 & -2.586277 \\
\hline C & 249 & -0.34 & -2.45 & -0.9 & -1.8 & -1.4 \\
\hline C & 90 & 9 & -2.4 & 0.2 & -3.0 & -0.8 \\
\hline C & 3.5 & 0.2 & -1.2 & 47 & 70 & -2 \\
\hline C & 3.509912 & 0.290418 & 1.26 & 110 & 353 & -3.316026 \\
\hline C & 2.923261 & -0.059599 & $0.024866 \mathrm{H}$ & -3.272110 & -0.625353 & -3.316026 \\
\hline C & -1.6 & & -0.0 & & 07 & \\
\hline lo & -2.851190 & -0.191319 & $61 \mathrm{H}$ & -4.540561 & -0.739420 & -1.18498 \\
\hline
\end{tabular}




\begin{tabular}{l|rrr|rrr|}
$\mathrm{C}$ & 1.648737 & -0.771545 & $-0.000521 \mathrm{H}$ & 0.937585 & 1.894181 & 0.803542 \\
$\mathrm{C}$ & 2.805016 & 0.152398 & $2.455099 \mathrm{H}$ & -0.292694 & 3.098827 & 1.202701 \\
$\mathrm{C}$ & -1.044444 & 1.068785 & $1.280182 \mathrm{H}$ & 0.417036 & 2.102873 & 2.471395 \\
$\mathrm{C}$ & -2.923261 & 0.059599 & $0.024866 \mathrm{H}$ & -4.486440 & -0.779362 & 1.253063 \\
$\mathrm{C}$ & -3.552118 & -0.276566 & $-1.214166 \mathrm{H}$ & -0.937585 & -1.894181 & 0.803542 \\
$\mathrm{C}$ & 1.044444 & -1.068785 & $1.280182 \mathrm{H}$ & 0.292694 & -3.098827 & 1.202701 \\
$\mathrm{C}$ & 1.527537 & -0.440112 & $2.462559 \mathrm{H}$ & -0.417036 & -2.102873 & 2.471395 \\
$\mathrm{C}$ & 0.069654 & 2.083832 & $1.436887 \mathrm{H}$ & 1.146211 & -0.249245 & 4.611769 \\
$\mathrm{C}$ & -1.527537 & 0.440112 & $2.462559 \mathrm{H}$ & -1.146211 & 0.249245 & 4.611769 \\
$\mathrm{C}$ & -3.509912 & -0.290418 & $1.262115 \mathrm{H}$ & -3.190602 & -0.596607 & 3.375483 \\
$\mathrm{C}$ & -0.069654 & -2.083832 & 1.436887 \\
$\mathrm{C}$ & 0.660668 & -0.187908 & 3.630447 \\
$\mathrm{C}$ & -0.660668 & 0.187908 & 3.630447 \\
$\mathrm{C}$ & -2.805016 & -0.152398 & 2.455099 & & & \\
\hline
\end{tabular}

$\sigma$-bonded 14 singlet dianion by $\mathrm{R}(\mathrm{U}) \mathrm{B} 3 \mathrm{LYP} / 6-31 \mathrm{G}^{*}, \mathrm{C}_{2}$ symmetry:

\begin{tabular}{|c|c|c|c|c|c|c|}
\hline & $x$ & $y$ & & $x$ & & \\
\hline C & -0.086475 & 0.682021 & $3.733907 \mathrm{H}$ & 0.557535 & 4.527785 & 1.210171 \\
\hline C & 0.086475 & -0.682021 & $3.733907 \mathrm{H}$ & 0.616457 & 4.503529 & -1.238580 \\
\hline C & 0.224724 & -1.443869 & $2.508422 \mathrm{H}$ & -0.071965 & 1.232153 & 075 \\
\hline C & 0.594031 & -0.648898 & $1.257971 \mathrm{H}$ & 071965 & -1.232153 & 075 \\
\hline C & 1.988107 & 0.003779 & $79 \mathrm{H}$ & 114925 & 0.964277 & 276 \\
\hline C & -0.594031 & 0.648898 & $1.257971 \mathrm{H}$ & 2.779274 & -0.678600 & 1.172631 \\
\hline C & -1.988107 & -0.003779 & $1.509479 \mathrm{H}$ & 2.130890 & 0.172024 & 2.578390 \\
\hline C & -0.224724 & 1.443869 & $2.508422 \mathrm{H}$ & -2.114925 & -0.964277 & 1.006276 \\
\hline C & 0.11 & 2.780687 & 2.42 & -2.7 & 0.6 & 631 \\
\hline C & 0.224724 & 3.489622 & 1.21 & -2.1 & -0.1 & 3390 \\
\hline C & 0.229634 & 3.482697 & -1.25 & 894 & 700 & 1943 \\
\hline C & -0.010548 & 2.830932 & -0.0 & -0.3 & -3.2 & 3.361943 \\
\hline C & 0.564145 & $-1.4 \varepsilon$ & -0.03 & 05 & 828 & 3696 \\
\hline C & -0.11 & $-2.7 \varepsilon$ & 2.4 & -0.5 & -4.5 & 171 \\
\hline C & -0.564145 & 1.48 & -0.03 & 969 & 559 & -1.036632 \\
\hline C & 0.101384 & 2.804900 & -2.45 & 2.9 & -0.2 & 7848 \\
\hline C & 0.967218 & -0.960629 & -1.28 & 2.22 & 338 & -2.525908 \\
\hline C & 0.010548 & -2.830932 & -0.01 & -0.6 & -4.5 & 8580 \\
\hline C & -0.224724 & -3.4 & 1.2 & 69 & $-1 .($ & 632 \\
\hline C & -0.967218 & 0.9 & $-1.2 \varepsilon$ & & 0.2 & 7848 \\
\hline C & -0.446248 & 1.510170 & $-2.503898 \mathrm{H}$ & -2.221675 & -0.262338 & -2.525908 \\
\hline C & 2.029341 & 0.118644 & -1.45 & -0.273638 & 1.142460 & -4.660456 \\
\hline C & 0.446248 & -1.5 & -2.50 & 638 & -1.1 & -4.660456 \\
\hline C & -0.229634 & -3.482697 & $-1.257696 \mathrm{H}$ & -0.466105 & -3.258828 & -3.383696 \\
\hline C & -2.029341 & -0.118644 & -1.459493 & & & \\
\hline C & -0.207826 & 0.653930 & -3.68 & & & \\
\hline C & 0.207826 & -0.653930 & -3.68 & & & \\
\hline C & -0.101384 & -2.804900 & -2.457796 & & & \\
\hline
\end{tabular}


Table 4S. Energies and vibrational frequencies of stationary points of 9, 13, and 14 .

\begin{tabular}{|c|c|c|c|c|c|}
\hline \multicolumn{6}{|c|}{$\sigma$-bonded Cope reactant 9 (singlet), $C_{2}$ symmetry, global minimum } \\
\hline \multicolumn{6}{|c|}{ RB3LYP/6-31G* and UB3LYP/6-31G* optimizations give the same results } \\
\hline \multicolumn{6}{|c|}{ Energy $=-1311.22243416$ Hartree $\quad$ Zero-point correction $=0.49573$} \\
\hline \multicolumn{6}{|c|}{ Thermal correction to Energy $=0.519978$ Hartree } \\
\hline \multicolumn{6}{|c|}{ Thermal correction to Enthalpy $=0.520922$ Hartree } \\
\hline \multicolumn{6}{|c|}{ Thermal correction to Gibbs Free Energy $=0.448115$ Hartree } \\
\hline \multicolumn{6}{|c|}{ Sum of electronic and zero-point Energies $=-1310.726704$ Hartree } \\
\hline \multicolumn{6}{|c|}{ Sum of electronic and thermal Energies = -1310.702457 Hartree } \\
\hline \multicolumn{6}{|c|}{ Sum of electronic and thermal Enthalpies $=-1310.701513$ Hartree } \\
\hline \multicolumn{6}{|c|}{ Sum of electronic and thermal Free Energies $=-1310.774319$ Hartree } \\
\hline & E (Thermal) & $\mathrm{CV}$ & $\mathrm{S}$ & & \\
\hline & $\mathrm{KCal} / \mathrm{Mol}$ & $\mathrm{Cal} / \mathrm{Mol}-\mathrm{K}$ & $\mathrm{Cal} / \mathrm{Mol}-\mathrm{K}$ & & \\
\hline TOTAL & 326.291 & 109.165 & 153.234 & & \\
\hline \multicolumn{6}{|c|}{ Frequencies $\left(\mathrm{cm}^{-1}\right)$} \\
\hline 49.6 & 81.9 & 90.5 & 129.4 & 160.6 & 163.7 \\
\hline 185.9 & 195.8 & 198.2 & 214.0 & 239.8 & 245.7 \\
\hline 251.7 & 276.7 & 281.8 & 284.4 & 295.5 & 304.9 \\
\hline 316.7 & 326.7 & 330.0 & 334.5 & 340.4 & 343.2 \\
\hline 364.3 & 381.5 & 381.7 & 388.5 & 397.7 & 414.6 \\
\hline 420.0 & 423.7 & 458.6 & 469.8 & 475.1 & 482.2 \\
\hline 495.0 & 530.0 & 531.1 & 532.8 & 563.2 & 569.0 \\
\hline 577.0 & 597.7 & 603.4 & 609.7 & 620.9 & 650.7 \\
\hline 652.0 & 660.7 & 679.2 & 684.1 & 698.7 & 708.9 \\
\hline 713.6 & 738.3 & 762.8 & 770.0 & 773.0 & 782.4 \\
\hline 788.8 & 795.4 & 810.3 & 812.4 & 818.4 & 830.1 \\
\hline 850.7 & 860.6 & 876.5 & 882.4 & 886.2 & 900.5 \\
\hline 905.6 & 915.3 & 923.0 & 930.4 & 952.0 & 962.0 \\
\hline 966.2 & 966.8 & 967.7 & 980.8 & 1028.7 & 1037.7 \\
\hline 1056.9 & 1062.1 & 1062.2 & 1066.5 & 1075.0 & 1085.1 \\
\hline 1088.3 & 1096.6 & 1112.3 & 1120.9 & 1153.3 & 1155.1 \\
\hline 1163.9 & 1178.7 & 1195.4 & 1207.6 & 1208.0 & 1220.5 \\
\hline 1220.9 & 1230.3 & 1235.0 & 1245.4 & 1267.1 & 1285.8 \\
\hline 1286.4 & 1291.7 & 1355.7 & 1356.3 & 1371.6 & 1377.6 \\
\hline 1389.3 & 1391.8 & 1400.1 & 1408.0 & 1428.0 & 1433.4 \\
\hline 1438.0 & 1439.0 & 1445.2 & 1449.4 & 1449.7 & 1455.8 \\
\hline 1476.8 & 1477.2 & 1505.5 & 1510.8 & 1518.2 & 1518.6 \\
\hline 1525.1 & 1525.5 & 1545.8 & 1546.5 & 1553.8 & 1553.9 \\
\hline 1592.5 & 1598.7 & 1619.4 & 1628.9 & 1633.2 & 1648.0 \\
\hline 1651.2 & 1655.9 & 1683.9 & 1696.5 & 3077.5 & 3077.6 \\
\hline 3087.0 & 3087.0 & 3142.6 & 3142.8 & 3145.2 & 3159.2 \\
\hline 3159.7 & 3160.4 & 3160.8 & 3164.9 & 3168.1 & 3169.0 \\
\hline 3169.1 & 3174.1 & 3174.4 & 3179.0 & 3179.0 & 3185.8 \\
\hline 3185.8 & 3186.7 & 3187.7 & 3187.7 & 3205.5 & 3205.7 \\
\hline
\end{tabular}




\begin{tabular}{|c|c|c|c|c|c|}
\hline \multicolumn{6}{|c|}{ Cope transition state of $9, C_{2}$ symmetry } \\
\hline \multicolumn{6}{|c|}{$\begin{array}{l}\text { RB3LYP/6-31G* and UB3LYP/6-31G* optimizations give the same results } \\
\text { Energy }=-1311.21233761 \text { Hartree } \\
\text { Zero-point correction }=0.493567 \text { Hartree } \\
\text { Thermal correction to Energy }=0.518050 \text { Hartree } \\
\text { Thermal correction to Enthalpy }=0.518994 \text { Hartree } \\
\text { Thermal correction to Gibbs Free Energy }=0.445330 \text { Hartree } \\
\text { Sum of electronic and zero-point Energies }=-1310.718770 \text { Hartree } \\
\text { Sum of electronic and thermal Energies }=-1310.694288 \text { Hartree } \\
\text { Sum of electronic and thermal Enthalpies }=-1310.693343 \text { Hartree } \\
\text { Sum of electronic and thermal Free Energies }=-1310.767007 \text { Hartree }\end{array}$} \\
\hline TOTAL & $\begin{array}{c}\text { E (Thermal) } \\
\text { KCal/Mol } \\
325.081 \\
\end{array}$ & $\begin{array}{c}\mathrm{CV} \\
\mathrm{Cal} / \mathrm{Mol}-\mathrm{K} \\
108.650 \\
\end{array}$ & $\begin{array}{c}\mathrm{S} \\
\text { Cal/Mol-K } \\
155.039 \\
\end{array}$ & & \\
\hline \multicolumn{6}{|c|}{ Frequencies $\left(\mathrm{cm}^{-1}\right)$} \\
\hline-276.3 & 52.1 & 70.4 & 93.0 & 118.7 & 134.3 \\
\hline 149.8 & 177.0 & 184.9 & 187.5 & 195.7 & 209.2 \\
\hline 231.3 & 245.5 & 247.7 & 272.9 & 285.0 & 290.9 \\
\hline 304.3 & 315.1 & 328.2 & 328.9 & 332.8 & 335.1 \\
\hline 335.9 & 362.0 & 378.9 & 380.8 & 382.4 & 392.1 \\
\hline 415.4 & 419.7 & 437.0 & 459.9 & 461.4 & 468.6 \\
\hline 474.4 & 501.4 & 527.1 & 527.2 & 533.4 & 560.8 \\
\hline 567.5 & 579.6 & 598.6 & 600.0 & 614.4 & 648.0 \\
\hline 652.0 & 657.1 & 667.7 & 674.6 & 685.3 & 693.1 \\
\hline 709.0 & 713.0 & 763.2 & 771.2 & 773.5 & 778.6 \\
\hline 782.0 & 796.6 & 808.2 & 810.3 & 816.1 & 821.9 \\
\hline 845.4 & 848.9 & 871.4 & 881.7 & 889.6 & 899.9 \\
\hline 902.9 & 905.9 & 915.3 & 921.6 & 964.6 & 966.4 \\
\hline 967.9 & 971.9 & 982.0 & 989.2 & 1021.3 & 1043.2 \\
\hline 1048.5 & 1054.5 & 1056.6 & 1061.7 & 1073.3 & 1084.1 \\
\hline 1086.5 & 1100.7 & 1123.6 & 1152.9 & 1154.2 & 1158.4 \\
\hline 1191.9 & 1198.4 & 1207.7 & 1211.5 & 1219.8 & 1227.6 \\
\hline 1232.0 & 1241.5 & 1249.9 & 1252.8 & 1286.0 & 1290.0 \\
\hline 1301.5 & 1303.0 & 1352.9 & 1361.9 & 1380.0 & 1382.7 \\
\hline 1395.2 & 1401.7 & 1408.7 & 1412.3 & 1421.9 & 1430.6 \\
\hline 1438.2 & 1438.5 & 1441.1 & 1449.8 & 1450.6 & 1452.6 \\
\hline 1474.5 & 1477.4 & 1503.4 & 1508.7 & 1512.8 & 1513.4 \\
\hline 1520.8 & 1521.8 & 1541.7 & 1542.4 & 1551.2 & 1551.2 \\
\hline 1577.6 & 1597.8 & 1610.9 & 1625.3 & 1625.8 & 1639.2 \\
\hline 1656.1 & 1660.5 & 1668.2 & 1673.1 & 3064.8 & 3064.9 \\
\hline 3080.9 & 3081.0 & 3137.8 & 3138.1 & 3147.8 & 3148.2 \\
\hline 3148.5 & 3158.7 & 3158.7 & 3161.6 & 3167.3 & 3168.4 \\
\hline 3169.5 & 3174.3 & 3174.8 & 3179.2 & 3179.2 & 3180.7 \\
\hline 3180.9 & 3182.3 & 3186.6 & 3186.6 & 3204.9 & 3205.0 \\
\hline
\end{tabular}




\begin{tabular}{|c|c|c|c|c|c|}
\hline \multicolumn{6}{|c|}{$\pi$-intermediate of 9 (singlet), $C_{2 h}$ symmetry } \\
\hline \multicolumn{6}{|c|}{$\begin{array}{l}\text { RB3LYP/6-31G* for singlet optimization and UB3LYP/6-31G* for triplet single point } \\
\text { Energy }(\text { singlet) }=-1311.21708504 \text { Hartree, E (triplet) }=-1311.20484365 \text { Hartree } \\
\text { Zero-point correction }=0.493016 \text { Hartree } \\
\text { Thermal correction to Energy }=0.518398 \text { Hartree } \\
\text { Thermal correction to Enthalpy }=0.519342 \text { Hartree } \\
\text { Thermal correction to Gibbs Free Energy }=0.443454 \text { Hartree } \\
\text { Sum of electronic and zero-point Energies }=-1310.724069 \text { Hartree } \\
\text { Sum of electronic and thermal Energies }=-1310.698687 \text { Hartree } \\
\text { Sum of electronic and thermal Enthalpies }=-1310.697743 \text { Hartree } \\
\text { Sum of electronic and thermal Free Energies }=-1310.773631 \text { Hartree }\end{array}$} \\
\hline TOTAL & $\begin{array}{l}\text { nal) } \\
\text { Aol } \\
99\end{array}$ & $\begin{array}{c}\text { CV } \\
\text { Cal/Mol-K } \\
110.839\end{array}$ & $\begin{array}{c}\mathrm{S} \\
\mathrm{Cal} / \mathrm{Mol}-\mathrm{K} \\
159.718\end{array}$ & & \\
\hline \multicolumn{6}{|c|}{ Frequencies $\left(\mathrm{cm}^{-1}\right)$} \\
\hline 52.8 & 59.1 & 88.5 & 94.3 & 117.9 & 131.2 \\
\hline 154.2 & 163.3 & 164.2 & 180.9 & 198.4 & 206.4 \\
\hline 206.4 & 234.7 & 258.1 & 274.5 & 281.2 & 285.3 \\
\hline 318.3 & 320.7 & 325.6 & 326.1 & 329.3 & 350.2 \\
\hline 351.9 & 366.5 & 375.2 & 376.2 & 376.3 & 397.0 \\
\hline 414.4 & 417.4 & 440.8 & 457.6 & 461.4 & 463.9 \\
\hline 471.7 & 498.6 & 520.3 & 526.9 & 533.5 & 537.9 \\
\hline 562.2 & 578.7 & 598.7 & 604.4 & 610.0 & 642.6 \\
\hline 649.2 & 668.2 & 669.1 & 673.4 & 685.9 & 688.3 \\
\hline 706.3 & 711.0 & 759.3 & 762.3 & 777.0 & 777.3 \\
\hline 782.4 & 796.5 & 803.7 & 810.5 & 812.7 & 817.6 \\
\hline 841.3 & 842.5 & 857.1 & 872.2 & 883.7 & 895.3 \\
\hline 897.4 & 897.8 & 903.6 & 903.7 & 965.4 & 965.8 \\
\hline 974.0 & 982.8 & 986.2 & 994.8 & 1029.4 & 1043.1 \\
\hline 1044.6 & 1050.9 & 1055.2 & 1058.3 & 1068.5 & 1082.5 \\
\hline 1087.6 & 1102.2 & 1124.3 & 1151.9 & 1158.9 & 1172.5 \\
\hline 1210.6 & 1213.3 & 1221.2 & 1222.2 & 1224.0 & 1230.0 \\
\hline 1233.2 & 1248.4 & 1254.5 & 1260.2 & 1290.1 & 1292.9 \\
\hline 1318.7 & 1322.4 & 1348.6 & 1365.9 & 1370.5 & 1372.1 \\
\hline 1395.5 & 1398.1 & 1412.3 & 1419.5 & 1419.8 & 1431.2 \\
\hline 1436.0 & 1436.3 & 1438.8 & 1448.0 & 1450.0 & 1452.8 \\
\hline 1489.9 & 1493.8 & 1497.8 & 1504.1 & 1505.7 & 1506.1 \\
\hline 1511.1 & 1512.3 & 1540.2 & 1540.3 & 1550.1 & 1551.1 \\
\hline 1568.4 & 1588.6 & 1592.8 & 1602.1 & 1619.3 & 1625.3 \\
\hline 1640.8 & 1645.7 & 1668.2 & 1668.3 & 3064.1 & 3064.2 \\
\hline 3077.1 & 3077.4 & 3120.9 & 3121.3 & 3134.9 & 3135.0 \\
\hline 3147.2 & 3147.2 & 3154.9 & 3155.0 & 3158.2 & 3158.3 \\
\hline 3166.9 & 3167.1 & 3176.1 & 3176.4 & 3176.4 & 3176.6 \\
\hline 3180.0 & 3180.1 & 3188.0 & 3188.0 & 3204.1 & 3204.3 \\
\hline
\end{tabular}




\begin{tabular}{|c|c|c|c|c|c|}
\hline \multicolumn{6}{|c|}{$\pi$-intermediate of 9 (singlet), $C_{2 h}$ symmetry } \\
\hline \multicolumn{6}{|c|}{$\begin{array}{l}\text { UB3LYP/6-31G* for singlet optimization, and the obtained geometry is used for triplet } \\
\text { single point at UB3LYP/6-31G* level of theory } \\
\text { Energy ( } \text { singlet) }=-1311.22072309 \text { Hartree, Energy (triplet) }=-1311.21453122 \text { Hartree } \\
\text { Zero-point correction }=0.491635 \text { Hartree } \\
\text { Thermal correction to Energy }=0.517205 \text { Hartree } \\
\text { Thermal correction to Enthalpy }=0.518150 \text { Hartree } \\
\text { Thermal correction to Gibbs Free Energy }=0.441787 \text { Hartree } \\
\text { Sum of electronic and zero-point Energies }=-1310.729088 \text { Hartree } \\
\text { Sum of electronic and thermal Energies }=-1310.703518 \text { Hartree } \\
\text { Sum of electronic and thermal Enthalpies }=-1310.702574 \text { Hartree } \\
\text { Sum of electronic and thermal Free Energies }=-1310.778936 \text { Hartree }\end{array}$} \\
\hline TOTAL & $\begin{array}{c}\text { E (Thermal) } \\
\text { KCal/Mol } \\
324.551\end{array}$ & $\begin{array}{c}\mathrm{CV} \\
\mathrm{Cal} / \mathrm{Mol}-\mathrm{K} \\
111.381\end{array}$ & $\begin{array}{c}\mathrm{S} \\
\mathrm{Cal} / \mathrm{Mol}-\mathrm{K} \\
160.719\end{array}$ & & \\
\hline \multicolumn{6}{|c|}{ Frequencies $\left(\mathrm{cm}^{-1}\right)$} \\
\hline 50.8 & 62.1 & 86.4 & 86.4 & 117.6 & 136.5 \\
\hline 143.9 & 165.2 & 168.2 & 173.3 & 180.2 & 181.1 \\
\hline 207.1 & 220.9 & 268.6 & 268.7 & 280.3 & 282.2 \\
\hline 314.5 & 323.3 & 325.3 & 327.8 & 342.0 & 354.9 \\
\hline 355.7 & 363.3 & 373.1 & 377.5 & 378.1 & 404.5 \\
\hline 412.3 & 413.8 & 438.8 & 453.4 & 461.8 & 462.3 \\
\hline 466.4 & 496.1 & 514.4 & 523.6 & 524.7 & 530.8 \\
\hline 557.1 & 574.0 & 597.1 & 598.8 & 608.5 & 635.8 \\
\hline 643.2 & 663.6 & 671.2 & 671.3 & 681.5 & 683.8 \\
\hline 701.6 & 707.3 & 749.5 & 756.4 & 768.8 & 774.3 \\
\hline 783.9 & 792.0 & 801.1 & 804.3 & 811.0 & 811.5 \\
\hline 836.3 & 837.7 & 846.7 & 866.0 & 873.6 & 885.7 \\
\hline 887.4 & 894.0 & 895.5 & 900.6 & 962.2 & 962.5 \\
\hline 972.2 & 985.4 & 988.3 & 995.5 & 1036.0 & 1040.0 \\
\hline 1040.6 & 1048.5 & 1054.6 & 1058.6 & 1062.9 & 1081.8 \\
\hline 1086.2 & 1100.2 & 1122.7 & 1149.3 & 1160.3 & 1171.5 \\
\hline 1209.2 & 1211.3 & 1215.4 & 1224.4 & 1224.8 & 1229.1 \\
\hline 1234.3 & 1247.1 & 1251.0 & 1263.6 & 1284.1 & 1288.9 \\
\hline 1306.7 & 1312.7 & 1331.1 & 1332.6 & 1352.9 & 1357.3 \\
\hline 1386.5 & 1388.2 & 1410.9 & 1420.8 & 1421.2 & 1432.3 \\
\hline 1435.9 & 1436.9 & 1437.5 & 1450.7 & 1451.2 & 1452.9 \\
\hline 1487.3 & 1496.9 & 1497.7 & 1501.0 & 1504.7 & 1504.8 \\
\hline 1508.1 & 1508.5 & 1540.5 & 1540.6 & 1552.2 & 1552.7 \\
\hline 1565.1 & 1569.3 & 1572.0 & 1579.6 & 1600.2 & 1603.2 \\
\hline 1614.4 & 1615.5 & 1661.9 & 1662.5 & 3066.4 & 3066.4 \\
\hline 3079.8 & 3079.9 & 3124.1 & 3124.3 & 3139.8 & 3139.8 \\
\hline 3139.8 & 3140.1 & 3152.2 & 3152.3 & 3156.4 & 3156.6 \\
\hline 3160.0 & 3160.2 & 3176.8 & 3176.9 & 3177.2 & 3177.3 \\
\hline 3180.6 & 3180.7 & 3188.8 & 3188.8 & 3204.5 & 3204.7 \\
\hline
\end{tabular}




\begin{tabular}{|c|c|c|c|c|c|}
\hline \multicolumn{6}{|c|}{ Aromatic intermediate of 9 (singlet), $C_{2 h}$ symmetry } \\
\hline \multirow{10}{*}{\multicolumn{6}{|c|}{$\begin{array}{l}\text { RB3LYP/6-31G* optimization } \\
\text { Energy }=-1311.18967390 \text { Hartree } \\
\text { Zero-point correction }=0.493666 \text { Hartree } \\
\text { Thermal correction to Energy }=0.518111 \text { Hartree } \\
\text { Thermal correction to Enthalpy }=0.519055 \text { Hartree } \\
\text { Thermal correction to Gibbs Free Energy }=0.445437 \text { Hartree } \\
\text { Sum of electronic and zero-point Energies }=-1310.696007 \text { Hartree } \\
\text { Sum of electronic and thermal Energies }=-1310.671563 \text { Hartree } \\
\text { Sum of electronic and thermal Enthalpies }=-1310.670619 \text { Hartree } \\
\text { Sum of electronic and thermal Free Energies }=-1310.744237 \text { Hartree }\end{array}$}} \\
\hline & & & & & \\
\hline & & & & & \\
\hline & & & & & \\
\hline & & & & & \\
\hline & & & & & \\
\hline & & & & & \\
\hline & & & & & \\
\hline & & & & & \\
\hline & & & & & \\
\hline \multicolumn{6}{|c|}{$\begin{array}{lll}\mathrm{E}(\text { Thermal }) & \mathrm{CV} & \mathrm{S}\end{array}$} \\
\hline \multicolumn{6}{|c|}{$\mathrm{KCal} / \mathrm{Mol}$} \\
\hline TOTAL & 325.119 & 109.435 & 154.941 & & \\
\hline \multicolumn{6}{|c|}{ Frequencies $\left(\mathrm{cm}^{-1}\right)$} \\
\hline 43.2 & 78.2 & 93.1 & 128.9 & 137.3 & 146.3 \\
\hline 163.2 & 179.4 & 186.5 & 194.1 & 217.2 & 230.0 \\
\hline 243.3 & 270.2 & 283.4 & 285.6 & 287.5 & 291.1 \\
\hline 311.3 & 317.0 & 317.4 & 320.2 & 336.9 & 346.4 \\
\hline 353.7 & 387.7 & 389.8 & 394.0 & 407.3 & 431.1 \\
\hline 433.5 & 435.9 & 482.3 & 486.0 & 488.5 & 512.9 \\
\hline 518.2 & 518.4 & 531.3 & 557.9 & 590.4 & 593.7 \\
\hline 597.8 & 608.7 & 619.7 & 630.7 & 643.7 & 645.5 \\
\hline 654.1 & 662.4 & 664.9 & 677.7 & 699.2 & 700.7 \\
\hline 702.8 & 750.9 & 764.7 & 769.6 & 773.7 & 774.4 \\
\hline 794.7 & 806.3 & 806.9 & 819.8 & 824.1 & 859.6 \\
\hline 864.1 & 864.8 & 876.4 & 889.2 & 894.7 & 911.6 \\
\hline 917.0 & 917.6 & 921.6 & 926.5 & 929.3 & 932.2 \\
\hline 936.9 & 946.9 & 965.5 & 966.0 & 966.5 & 996.6 \\
\hline 1009.8 & 1014.0 & 1015.1 & 1046.1 & 1073.2 & 1075.7 \\
\hline 1080.6 & 1087.7 & 1090.4 & 1106.6 & 1120.6 & 1130.0 \\
\hline 1145.2 & 1171.5 & 1192.4 & 1193.3 & 1194.4 & 1206.5 \\
\hline 1209.8 & 1224.0 & 1245.0 & 1248.1 & 1255.3 & 1289.3 \\
\hline 1295.7 & 1312.0 & 1324.1 & 1334.4 & 1343.5 & 1370.1 \\
\hline 1384.2 & 1389.4 & 1392.9 & 1393.7 & 1403.4 & 1417.5 \\
\hline 1420.2 & 1420.3 & 1431.8 & 1433.0 & 1433.4 & 1458.5 \\
\hline 1485.0 & 1493.5 & 1500.1 & 1503.7 & 1510.8 & 1512.5 \\
\hline 1517.4 & 1518.4 & 1532.7 & 1534.7 & 1543.0 & 1546.4 \\
\hline 1553.7 & 1559.8 & 1566.7 & 1572.9 & 1602.7 & 1620.3 \\
\hline 1622.1 & 1631.2 & 1667.0 & 1671.7 & 3085.8 & 3086.1 \\
\hline 3095.7 & 3095.9 & 3161.0 & 3161.2 & 3161.2 & 3161.5 \\
\hline 3161.5 & 3161.8 & 3164.2 & 3164.7 & 3167.4 & 3167.6 \\
\hline 3176.3 & 3176.6 & 3177.7 & 3178.2 & 3183.8 & 3184.3 \\
\hline 3184.5 & 3184.7 & 3199.0 & 3199.2 & 3204.9 & 3205.1 \\
\hline
\end{tabular}




\begin{tabular}{|c|c|c|c|c|c|}
\hline \multicolumn{6}{|c|}{ Aromatic intermediate of 9 (singlet), $C_{2 h}$ symmetry } \\
\hline \multicolumn{6}{|c|}{ UB3LYP/6-31G* optimization } \\
\hline \multicolumn{6}{|c|}{ Energy $=-1311.20087098$ Hartree } \\
\hline \multicolumn{6}{|c|}{ Zero-point correction $=0.492835$ Hartree } \\
\hline \multicolumn{6}{|c|}{ Thermal correction to Energy $=0.517265$ Hartree } \\
\hline \multicolumn{6}{|c|}{ Thermal correction to Enthalpy $=0.518209$ Hartree } \\
\hline \multicolumn{6}{|c|}{ Thermal correction to Gibbs Free Energy $=0.444527$ Hartree } \\
\hline \multicolumn{6}{|c|}{ Sum of electronic and zero-point Energies $=-1310.708036$ Hartree } \\
\hline \multicolumn{6}{|c|}{ Sum of electronic and thermal Energies $=-1310.683606$ Hartree } \\
\hline \multicolumn{6}{|c|}{ Sum of electronic and thermal Enthalpies $=-1310.682662$ Hartree } \\
\hline \multicolumn{6}{|c|}{ Sum of electronic and thermal Free Energies $=-1310.756344$ Hartree } \\
\hline & E (Thermal) & $\mathrm{CV}$ & $\mathrm{S}$ & & \\
\hline & $\mathrm{KCal} / \mathrm{Mol}$ & $\mathrm{Cal} / \mathrm{Mol}-\mathrm{K}$ & $\mathrm{Cal} / \mathrm{Mol}-\mathrm{K}$ & & \\
\hline TOTAL & 324.588 & 109.335 & 155.076 & & \\
\hline \multicolumn{6}{|c|}{ Frequencies $\left(\mathrm{cm}^{-1}\right)$} \\
\hline 42.3 & 78.5 & 83.5 & 135.6 & 139.8 & 144.5 \\
\hline 164.6 & 173.8 & 178.3 & 194.1 & 201.1 & 236.0 \\
\hline 252.3 & 263.1 & 284.4 & 286.7 & 287.7 & 293.4 \\
\hline 311.0 & 313.9 & 314.3 & 333.9 & 350.1 & 353.0 \\
\hline 379.5 & 383.5 & 392.6 & 395.1 & 401.7 & 415.5 \\
\hline 425.5 & 428.2 & 480.7 & 482.3 & 487.3 & 503.9 \\
\hline 516.4 & 517.9 & 556.0 & 560.6 & 577.0 & 599.8 \\
\hline 604.3 & 608.4 & 610.7 & 618.6 & 641.7 & 644.9 \\
\hline 645.3 & 657.3 & 659.0 & 701.9 & 704.1 & 706.5 \\
\hline 732.0 & 748.2 & 761.4 & 767.3 & 770.4 & 785.0 \\
\hline 794.2 & 797.4 & 814.0 & 821.9 & 849.3 & 861.5 \\
\hline 863.6 & 879.6 & 889.9 & 895.0 & 895.2 & 906.1 \\
\hline 915.2 & 918.8 & 927.8 & 931.5 & 947.6 & 952.3 \\
\hline 953.7 & 956.4 & 964.6 & 967.0 & 981.6 & 1011.6 \\
\hline 1026.4 & 1039.0 & 1042.4 & 1050.8 & 1061.9 & 1075.6 \\
\hline 1084.3 & 1090.7 & 1096.0 & 1112.8 & 1120.8 & 1133.6 \\
\hline 1164.5 & 1169.0 & 1169.2 & 1169.4 & 1172.7 & 1186.8 \\
\hline 1196.8 & 1197.7 & 1199.2 & 1241.4 & 1243.3 & 1277.2 \\
\hline 1282.2 & 1287.4 & 1294.5 & 1303.1 & 1339.4 & 1343.1 \\
\hline 1347.1 & 1377.6 & 1382.7 & 1396.7 & 1405.5 & 1408.0 \\
\hline 1419.4 & 1419.8 & 1430.7 & 1431.5 & 1435.6 & 1441.4 \\
\hline 1442.8 & 1458.6 & 1464.9 & 1492.1 & 1508.7 & 1511.1 \\
\hline 1516.7 & 1517.6 & 1523.5 & 1537.6 & 1537.8 & 1538.7 \\
\hline 1551.5 & 1553.2 & 1565.4 & 1588.1 & 1612.8 & 1613.5 \\
\hline 1622.4 & 1627.9 & 1648.0 & 1657.0 & 3082.0 & 3082.1 \\
\hline 3093.6 & 3093.7 & 3157.4 & 3157.9 & 3159.7 & 3159.8 \\
\hline 3160.0 & 3160.2 & 3163.3 & 3163.9 & 3166.9 & 3167.1 \\
\hline 3169.2 & 3169.5 & 3177.8 & 3177.9 & 3183.1 & 3183.1 \\
\hline 3184.8 & 3184.9 & 3189.1 & 3189.8 & 3206.8 & 3206.9 \\
\hline
\end{tabular}




\begin{tabular}{|c|c|c|c|c|c|}
\hline \multicolumn{6}{|c|}{$\pi$-bonded structure of 13 (singlet), $C_{2 h}$ symmetry, second-order saddle point } \\
\hline \multicolumn{6}{|c|}{$\begin{array}{l}\text { RB3LYP/6-31G* for singlet optimization and UB3LYP/6-31G* for triplet single point } \\
\text { Energy }(\text { singlet) }=-1313.66821688 \text { Hartree, Energy }(\text { triplet })=-1313.66140542 \text { Hartree } \\
\text { Zero-point correction }=0.541758 \text { Hartree } \\
\text { Thermal correction to Energy }=0.566259 \text { Hartree } \\
\text { Thermal correction to Enthalpy }=0.567203 \text { Hartree } \\
\text { Thermal correction to Gibbs Free Energy }=0.493131 \text { Hartree } \\
\text { Sum of electronic and zero-point Energies }=-1313.126459 \text { Hartree } \\
\text { Sum of electronic and thermal Energies }=-1313.101958 \text { Hartree } \\
\text { Sum of electronic and thermal Enthalpies }=-1313.101014 \text { Hartree } \\
\text { Sum of electronic and thermal Free Energies }=-1313.175086 \text { Hartree }\end{array}$} \\
\hline TOTAL & $\begin{array}{c}\text { E (Thermal) } \\
\text { KCal/Mol } \\
355.333\end{array}$ & $\begin{array}{c}\mathrm{CV} \\
\mathrm{Cal} / \mathrm{Mol}-\mathrm{K} \\
109.461\end{array}$ & $\begin{array}{c}\mathrm{S} \\
\mathrm{Cal} / \mathrm{Mol}-\mathrm{K} \\
155.89\end{array}$ & & \\
\hline \multicolumn{6}{|c|}{ Frequencies $\left(\mathrm{cm}^{-1}\right)$} \\
\hline-172.1 & -171.9 & 53.2 & 60.2 & 77.7 & 101.9 \\
\hline 130.0 & 142.9 & 155.8 & 171.9 & 172.5 & 181.9 \\
\hline 209.1 & 214.5 & 274.3 & 282.3 & 284.5 & 288.4 \\
\hline 316.8 & 317.9 & 318.5 & 322.5 & 325.1 & 332.9 \\
\hline 340.9 & 365.3 & 369.3 & 389.7 & 398.7 & 405.6 \\
\hline 408.7 & 426.3 & 426.6 & 448.3 & 458.6 & 474.9 \\
\hline 476.0 & 502.8 & 509.7 & 527.2 & 528.2 & 538.6 \\
\hline 568.1 & 575.3 & 581.1 & 581.7 & 601.3 & 615.1 \\
\hline 622.8 & 650.6 & 661.7 & 664.4 & 667.4 & 672.6 \\
\hline 676.3 & 695.4 & 719.2 & 726.0 & 771.3 & 772.4 \\
\hline 777.2 & 797.8 & 803.7 & 809.3 & 813.0 & 819.6 \\
\hline 845.2 & 849.1 & 854.5 & 876.0 & 883.4 & 885.6 \\
\hline 886.4 & 888.2 & 899.3 & 904.3 & 923.7 & 933.0 \\
\hline 963.8 & 964.0 & 984.7 & 990.4 & 1019.9 & 1029.7 \\
\hline 1034.0 & 1034.1 & 1043.7 & 1046.2 & 1051.9 & 1062.1 \\
\hline 1069.7 & 1079.6 & 1096.4 & 1097.0 & 1131.4 & 1133.0 \\
\hline 1163.7 & 1165.5 & 1191.8 & 1194.4 & 1212.1 & 1218.9 \\
\hline 1227.4 & 1230.5 & 1234.8 & 1235.7 & 1243.6 & 1246.4 \\
\hline 1268.6 & 1273.5 & 1285.5 & 1286.8 & 1314.3 & 1316.6 \\
\hline 1334.7 & 1337.5 & 1345.7 & 1359.2 & 1372.4 & 1383.1 \\
\hline 1384.5 & 1390.7 & 1392.7 & 1397.4 & 1427.9 & 1429.7 \\
\hline 1433.8 & 1435.5 & 1441.9 & 1444.5 & 1453.4 & 1454.9 \\
\hline 1493.3 & 1493.7 & 1495.6 & 1499.2 & 1502.1 & 1503.9 \\
\hline 1513.7 & 1514.6 & 1524.5 & 1529.5 & 1537.9 & 1540.4 \\
\hline 1542.3 & 1546.8 & 1567.8 & 1572.3 & 1576.7 & 1594.1 \\
\hline 1601.3 & 1604.3 & 1627.4 & 1628.5 & 1641.1 & 1647.8 \\
\hline 3060.9 & 3061.0 & 3070.3 & 3070.8 & 3078.9 & 3079.5 \\
\hline 3090.1 & 3091.4 & 3094.3 & 3097.1 & 3111.2 & 3112.1 \\
\hline 3133.6 & 3133.9 & 3149.5 & 3149.8 & 3172.0 & 3172.0 \\
\hline 3172.1 & 3172.1 & 3177.7 & 3177.7 & 3182.9 & 3183.1 \\
\hline 3185.1 & 3185.3 & 3186.4 & 3186.4 & 3202.2 & 3202.5 \\
\hline
\end{tabular}




\begin{tabular}{|c|c|c|c|c|c|}
\hline \multicolumn{6}{|c|}{$\pi$-bonded structure of 13 (singlet), $C_{2 h}$ symmetry, second-order saddle point } \\
\hline \multicolumn{6}{|c|}{ UB3LYP/6-31G* optimization } \\
\hline \multicolumn{6}{|c|}{ Energy $=-1313.67444294$ Hartree } \\
\hline \multicolumn{6}{|c|}{ Zero-point correction $=0.539768$ Hartree } \\
\hline \multicolumn{6}{|c|}{ Thermal correction to Energy $=0.564853$ Hartree } \\
\hline \multicolumn{6}{|c|}{ Thermal correction to Enthalpy $=0.565798$ Hartree } \\
\hline \multicolumn{6}{|c|}{ Thermal correction to Gibbs Free Energy $=0.489543$ Hartree } \\
\hline \multicolumn{6}{|c|}{ Sum of electronic and zero-point Energies $=-1313.134675$ Hartree } \\
\hline \multicolumn{6}{|c|}{ Sum of electronic and thermal Energies $=-1313.109590$ Hartree } \\
\hline \multicolumn{6}{|c|}{ Sum of electronic and thermal Enthalpies $=-1313.108645$ Hartree } \\
\hline \multicolumn{6}{|c|}{ Sum of electronic and thermal Free Energies $=-1313.184900$ Hartree } \\
\hline \multicolumn{2}{|c|}{$\begin{array}{l}\text { E (Thermal) } \\
\text { KCal/Mol }\end{array}$} & $\mathrm{CV}$ & & & \\
\hline \multirow[b]{2}{*}{ Total } & Mol & Cal/Mol-K & & & \\
\hline & & 110.311 & & & \\
\hline \multicolumn{6}{|c|}{ Frequencies $\left(\mathrm{cm}^{-1}\right)$} \\
\hline-117.0 & -116.6 & 50.2 & 53.9 & 57.3 & 77.7 \\
\hline 94.0 & 102.6 & 121.6 & 148.3 & 148.3 & 156.1 \\
\hline 190.2 & 223.1 & 271.4 & 274.3 & 282.6 & 286.7 \\
\hline 307.8 & 314.1 & 316.3 & 324.6 & 328.2 & 332.4 \\
\hline 353.4 & 360.2 & 363.6 & 395.1 & 396.2 & 404.4 \\
\hline 413.6 & 419.8 & 431.7 & 446.2 & 453.4 & 475.9 \\
\hline 480.6 & 490.4 & 503.4 & 523.4 & 524.4 & 532.1 \\
\hline 565.9 & 570.4 & 573.0 & 573.6 & 596.3 & 613.4 \\
\hline 617.0 & 651.0 & 658.8 & 661.9 & 667.3 & 667.6 \\
\hline 677.4 & 690.4 & 694.0 & 710.8 & 764.1 & 766.9 \\
\hline 771.2 & 792.9 & 802.9 & 804.3 & 805.1 & 820.5 \\
\hline 838.2 & 840.2 & 850.4 & 868.2 & 878.7 & 880.9 \\
\hline 882.6 & 882.8 & 902.0 & 903.4 & 918.0 & 930.0 \\
\hline 960.1 & 960.3 & 984.1 & 986.2 & 1011.1 & 1029.5 \\
\hline 1029.6 & 1035.9 & 1039.9 & 1044.7 & 1052.2 & 1055.3 \\
\hline 1067.9 & 1074.4 & 1094.2 & 1094.6 & 1129.5 & 1131.0 \\
\hline 1157.0 & 1161.9 & 1180.0 & 1180.9 & 1207.8 & 1216.8 \\
\hline 1219.3 & 1227.1 & 1230.0 & 1236.3 & 1240.0 & 1245.2 \\
\hline 1267.5 & 1268.1 & 1276.5 & 1278.1 & 1298.6 & 1301.1 \\
\hline 1317.1 & 1319.3 & 1340.1 & 1343.4 & 1368.2 & 1378.0 \\
\hline 1383.1 & 1390.0 & 1390.3 & 1391.9 & 1428.0 & 1428.1 \\
\hline 1431.5 & 1432.0 & 1438.7 & 1439.3 & 1450.4 & 1452.3 \\
\hline 1491.2 & 1493.6 & 1499.2 & 1502.0 & 1505.7 & 1505.7 \\
\hline 1507.0 & 1508.0 & 1522.1 & 1526.1 & 1537.6 & 1545.9 \\
\hline 1546.7 & 1547.1 & 1573.4 & 1573.7 & 1573.9 & 1577.6 \\
\hline 1579.4 & 1581.6 & 1602.7 & 1603.3 & 1611.0 & 1611.9 \\
\hline 3056.2 & 3056.2 & 3065.7 & 3066.0 & 3081.4 & 3081.5 \\
\hline 3096.4 & 3097.3 & 3108.8 & 3110.5 & 3126.9 & 3128.3 \\
\hline 3137.3 & 3137.4 & 3158.4 & 3158.5 & 3170.3 & 3170.5 \\
\hline 3170.6 & 3170.7 & 3173.6 & 3174.1 & 3176.4 & 3177.1 \\
\hline 3178.6 & 3178.6 & 3187.2 & 3187.2 & 3203.2 & 3203.3 \\
\hline
\end{tabular}




\begin{tabular}{|c|c|c|c|c|c|}
\hline \multicolumn{6}{|c|}{$\sigma$-bonded structure of 13 (singlet), $C_{2}$ symmetry } \\
\hline \multicolumn{6}{|c|}{ RB3LYP/6-31G* and UB3LYP/6-31G* optimizations give the same results } \\
\hline \multicolumn{6}{|c|}{ Energy $=-1313.66409784$ Hartree } \\
\hline \multicolumn{6}{|c|}{ Zero-point correction $=0.545425$ Hartree } \\
\hline \multicolumn{6}{|c|}{ Thermal correction to Energy $=0.570057$ Hartree } \\
\hline \multicolumn{6}{|c|}{ Thermal correction to Enthalpy $=0.571001$ Hartree } \\
\hline \multicolumn{6}{|c|}{ Thermal correction to Gibbs Free Energy $=0.497389$ Hartree } \\
\hline \multicolumn{6}{|c|}{ Sum of electronic and zero-point Energies $=-1313.118673$ Hartree } \\
\hline \multicolumn{6}{|c|}{ Sum of electronic and thermal Energies $=-1313.094041$ Hartree } \\
\hline \multicolumn{6}{|c|}{ Sum of electronic and thermal Enthalpies $=-1313.093097$ Hartree } \\
\hline \multicolumn{6}{|c|}{ Sum of electronic and thermal Free Energies $=-1313.166709$ Hartree } \\
\hline & E (Thermal) & $\mathrm{CV}$ & $\mathrm{S}$ & & \\
\hline & $\mathrm{KCal} / \mathrm{Mol}$ & $\mathrm{Cal} / \mathrm{Mol}-\mathrm{K}$ & $\mathrm{Cal} / \mathrm{Mol}-\mathrm{K}$ & & \\
\hline TOTAL & 357.716 & 111.492 & 154.931 & & \\
\hline \multicolumn{6}{|c|}{ Frequencies $\left(\mathrm{cm}^{-1}\right)$} \\
\hline 55.4 & 65.9 & 83.9 & 128.5 & 134.7 & 152.2 \\
\hline 161.2 & 188.7 & 206.3 & 208.5 & 235.4 & 270.0 \\
\hline 280.5 & 284.4 & 287.8 & 289.4 & 296.3 & 307.4 \\
\hline 310.4 & 324.9 & 328.6 & 331.6 & 338.5 & 350.2 \\
\hline 364.3 & 376.6 & 386.4 & 403.8 & 405.0 & 409.3 \\
\hline 424.1 & 430.0 & 435.2 & 466.6 & 472.1 & 481.0 \\
\hline 489.8 & 515.7 & 529.4 & 535.1 & 535.3 & 559.7 \\
\hline 570.2 & 580.4 & 585.0 & 599.2 & 599.6 & 617.8 \\
\hline 628.2 & 653.4 & 653.5 & 663.6 & 667.1 & 680.1 \\
\hline 696.0 & 717.2 & 726.3 & 753.6 & 769.3 & 783.2 \\
\hline 784.0 & 800.0 & 801.5 & 814.8 & 822.6 & 826.8 \\
\hline 863.5 & 869.0 & 870.3 & 876.1 & 889.2 & 892.2 \\
\hline 904.7 & 911.5 & 916.3 & 918.8 & 926.2 & 937.3 \\
\hline 962.3 & 964.4 & 965.1 & 976.3 & 1025.4 & 1036.8 \\
\hline 1040.5 & 1050.7 & 1057.7 & 1063.7 & 1065.3 & 1071.5 \\
\hline 1079.9 & 1088.7 & 1090.3 & 1093.3 & 1114.4 & 1127.4 \\
\hline 1133.6 & 1158.5 & 1174.1 & 1181.4 & 1188.9 & 1205.2 \\
\hline 1205.4 & 1212.8 & 1217.8 & 1231.5 & 1236.6 & 1243.3 \\
\hline 1247.9 & 1251.4 & 1269.3 & 1274.8 & 1287.3 & 1288.6 \\
\hline 1329.0 & 1334.9 & 1364.2 & 1364.6 & 1369.7 & 1376.5 \\
\hline 1378.6 & 1389.6 & 1398.5 & 1399.7 & 1413.8 & 1414.7 \\
\hline 1439.4 & 1442.7 & 1452.3 & 1453.5 & 1453.8 & 1456.7 \\
\hline 1476.6 & 1477.3 & 1506.0 & 1506.3 & 1515.2 & 1519.1 \\
\hline 1522.7 & 1527.8 & 1529.1 & 1532.6 & 1533.2 & 1537.0 \\
\hline 1555.1 & 1558.3 & 1579.9 & 1581.2 & 1597.4 & 1603.0 \\
\hline 1640.3 & 1641.4 & 1653.0 & 1655.7 & 1710.5 & 1717.0 \\
\hline 3047.8 & 3048.3 & 3059.1 & 3068.4 & 3083.5 & 3083.6 \\
\hline 3084.7 & 3093.2 & 3095.5 & 3101.0 & 3105.0 & 3115.8 \\
\hline 3157.4 & 3157.5 & 3166.6 & 3166.7 & 3167.5 & 3167.7 \\
\hline 3169.5 & 3169.6 & 3176.1 & 3176.1 & 3178.8 & 3179.2 \\
\hline 3183.0 & 3183.0 & 3203.2 & 3203.3 & 3211.7 & 3211.7 \\
\hline
\end{tabular}




\begin{tabular}{|c|c|c|c|c|c|}
\hline \multicolumn{6}{|c|}{14 (neutral, singlet), $C_{2 h}$ symmetry } \\
\hline \multicolumn{6}{|c|}{$\begin{array}{l}\text { RB3LYP/6-31G* and UB3LYP/6-31G* optimizations give the same re } \\
\text { Energy }=-1081.37901249 \text { Hartree } \\
\text { Zero-point correction }=0.435259 \text { Hartree } \\
\text { Thermal correction to Energy }=0.456869 \text { Hartree } \\
\text { Thermal correction to Enthalpy }=0.457813 \text { Hartree } \\
\text { Thermal correction to Gibbs Free Energy }=0.389803 \text { Hartree } \\
\text { Sum of electronic and zero-point Energies }=-1080.943754 \text { Hartree } \\
\text { Sum of electronic and thermal Energies }=-1080.922144 \text { Hartree } \\
\text { Sum of electronic and thermal Enthalpies }=-1080.921200 \text { Hartree } \\
\text { Sum of electronic and thermal Free Energies }=-1080.989209 \text { Hartree }\end{array}$} \\
\hline Total & $\begin{array}{l}\text { E (Thermal) } \\
\text { KCal/Mol } \\
286.689\end{array}$ & $\begin{array}{c}\text { CV } \\
\text { Cal/Mol-K } \\
94.243\end{array}$ & & & \\
\hline \multicolumn{6}{|c|}{ Frequencies $\left(\mathrm{cm}^{-1}\right)$} \\
\hline 73.1 & 89.6 & 91.6 & 124.2 & 126.7 & 153.0 \\
\hline 166.5 & 192.7 & 204.4 & 208.3 & 214.3 & 241.8 \\
\hline 259.9 & 265.6 & 272.2 & 279.6 & 320.3 & 331.0 \\
\hline 342.8 & 355.7 & 366.8 & 369.9 & 395.2 & 395.3 \\
\hline 410.2 & 415.3 & 441.9 & 453.0 & 461.2 & 480.5 \\
\hline 486.9 & 491.4 & 491.9 & 524.9 & 538.5 & 558.4 \\
\hline 584.9 & 588.0 & 596.9 & 599.8 & 606.8 & 612.5 \\
\hline 659.6 & 692.7 & 694.7 & 700.6 & 718.4 & 755.8 \\
\hline 757.0 & 769.1 & 776.0 & 795.8 & 795.9 & 796.5 \\
\hline 810.1 & 812.4 & 820.5 & 848.9 & 859.4 & 885.1 \\
\hline 925.9 & 947.1 & 949.0 & 949.4 & 958.5 & 959.8 \\
\hline 989.7 & 989.9 & 997.7 & 1017.7 & 1048.0 & 1053.0 \\
\hline 1059.9 & 1061.7 & 1072.4 & 1073.1 & 1125.3 & 1132.2 \\
\hline 1147.6 & 1153.7 & 1186.5 & 1189.9 & 1195.8 & 1196.9 \\
\hline 1198.3 & 1204.3 & 1227.5 & 1233.2 & 1245.7 & 1256.5 \\
\hline 1265.6 & 1267.6 & 1344.8 & 1349.8 & 1360.4 & 1363.5 \\
\hline 1375.5 & 1380.5 & 1434.1 & 1436.8 & 1438.5 & 1442.2 \\
\hline 1443.4 & 1445.6 & 1451.8 & 1453.9 & 1479.6 & 1484.5 \\
\hline 1507.1 & 1507.4 & 1510.6 & 1510.7 & 1540.3 & 1540.6 \\
\hline 1547.3 & 1548.5 & 1549.3 & 1551.4 & 1585.1 & 1585.2 \\
\hline 1637.7 & 1642.1 & 1648.5 & 1650.8 & 1659.5 & 1662.9 \\
\hline 3073.5 & 3073.6 & 3086.9 & 3087.1 & 3127.6 & 3127.8 \\
\hline 3137.0 & 3137.1 & 3148.5 & 3148.7 & 3148.9 & 3149.0 \\
\hline 3154.8 & 3155.0 & 3167.1 & 3167.1 & 3175.2 & 3175.3 \\
\hline 3177.2 & 3177.2 & 3193.6 & 3193.6 & 3196.5 & 3196.7 \\
\hline
\end{tabular}




\begin{tabular}{|c|c|c|c|c|c|}
\hline \multicolumn{6}{|c|}{$\pi$-bonded structure of $14^{2+}$ (singlet), $C_{2 h}$ symmetry } \\
\hline \multicolumn{6}{|c|}{$\begin{array}{l}\text { RB3LYP/6-31G* and UB3LYP/6-31G* optimizations give the same } \mathrm{r} \\
\text { Energy }=-1080.79506691 \text { Hartree } \\
\text { Zero-point correction }=0.434702 \text { Hartree } \\
\text { Thermal correction to Energy }=0.456725 \text { Hartree } \\
\text { Thermal correction to Enthalpy }=0.457669 \text { Hartree } \\
\text { Thermal correction to Gibbs Free Energy }=0.388844 \text { Hartree } \\
\text { Sum of electronic and zero-point Energies }=-1080.360365 \text { Hartree } \\
\text { Sum of electronic and thermal Energies }=-1080.338342 \text { Hartree } \\
\text { Sum of electronic and thermal Enthalpies }=-1080.337398 \text { Hartree } \\
\text { Sum of electronic and thermal Free Energies }=-1080.406223 \text { Hartree }\end{array}$} \\
\hline & $\begin{array}{l}\text { rmal) } \\
\text { Mol } \\
599 \\
\end{array}$ & $\begin{array}{c}\text { CV } \\
\text { Cal/Mol-K } \\
94.857 \\
\end{array}$ & $\begin{array}{l}1-K \\
55\end{array}$ & & \\
\hline \multicolumn{6}{|c|}{ Frequencies $\left(\mathrm{cm}^{-1}\right)$} \\
\hline 72.1 & 87.7 & 99.0 & 126.1 & 135.1 & 155.6 \\
\hline 173.5 & 175.2 & 177.9 & 193.4 & 211.5 & 237.3 \\
\hline 242.7 & 247.3 & 263.8 & 270.2 & 304.5 & 327.0 \\
\hline 329.4 & 332.4 & 353.8 & 357.3 & 359.9 & 362.3 \\
\hline 393.1 & 397.2 & 407.1 & 425.9 & 445.0 & 466.3 \\
\hline 470.2 & 476.3 & 482.6 & 525.8 & 537.2 & 556.1 \\
\hline 562.1 & 570.7 & 583.9 & 598.5 & 613.7 & 621.7 \\
\hline 650.9 & 693.3 & 701.2 & 702.1 & 725.1 & 759.6 \\
\hline 760.4 & 769.4 & 781.5 & 785.8 & 790.8 & 800.7 \\
\hline 812.4 & 850.0 & 856.5 & 888.2 & 890.7 & 903.5 \\
\hline 918.5 & 932.7 & 984.8 & 993.4 & 996.6 & 999.3 \\
\hline 1006.2 & 1012.6 & 1015.3 & 1018.7 & 1025.0 & 1034.4 \\
\hline 1048.4 & 1049.9 & 1053.6 & 1054.3 & 1130.9 & 1140.5 \\
\hline 1155.1 & 1160.8 & 1182.5 & 1197.4 & 1197.5 & 1202.4 \\
\hline 1213.8 & 1243.5 & 1246.3 & 1248.1 & 1249.7 & 1251.1 \\
\hline 1280.8 & 1282.0 & 1326.8 & 1358.7 & 1361.8 & 1362.6 \\
\hline 1370.1 & 1385.4 & 1392.0 & 1392.4 & 1427.6 & 1429.0 \\
\hline 1439.4 & 1439.5 & 1448.1 & 1451.7 & 1460.8 & 1466.7 \\
\hline 1483.8 & 1491.6 & 1499.2 & 1500.3 & 1509.9 & 1522.2 \\
\hline 1523.9 & 1524.4 & 1530.2 & 1546.1 & 1547.3 & 1547.8 \\
\hline 1568.2 & 1591.5 & 1604.0 & 1609.8 & 1629.8 & 1636.4 \\
\hline 3070.6 & 3071.0 & 3076.8 & 3077.8 & 3126.3 & 3126.6 \\
\hline 3129.3 & 3129.4 & 3183.1 & 3183.1 & 3185.9 & 3186.1 \\
\hline 3186.3 & 3186.7 & 3196.2 & 3196.3 & 3211.0 & 3211.1 \\
\hline 3213.1 & 3213.2 & 3223.3 & 3223.3 & 3224.8 & 3224.8 \\
\hline
\end{tabular}




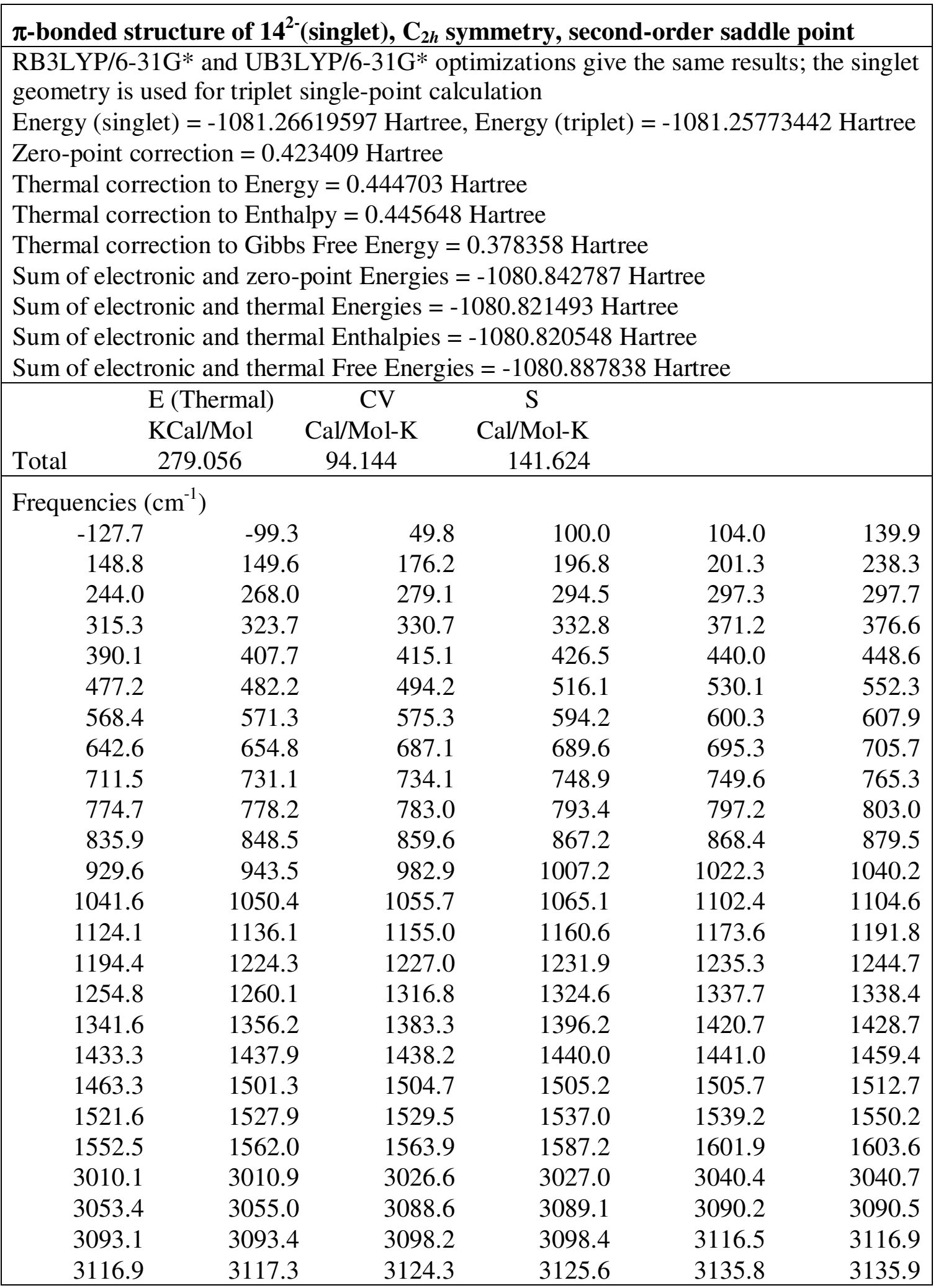




\begin{tabular}{|c|c|c|c|c|c|}
\hline \multicolumn{6}{|c|}{$\pi$-bonded structure of $14^{2-}$ with distortion (singlet), $C_{2}$ symmetry } \\
\hline \multicolumn{6}{|c|}{$\begin{array}{l}\text { RB3LYP/6-31G* and UB3LYP/6-31G* optimizations give the same re } \\
\text { Energy }=-1081.26707901 \text { Hartree } \\
\text { Zero-point correction }=0.424321 \text { Hartree } \\
\text { Thermal correction to Energy }=0.446761 \text { Hartree } \\
\text { Thermal correction to Enthalpy }=0.447705 \text { Hartree } \\
\text { Thermal correction to Gibbs Free Energy }=0.378251 \text { Hartree } \\
\text { Sum of electronic and zero-point Energies }=-1080.842758 \text { Hartree } \\
\text { Sum of electronic and thermal Energies }=-1080.820318 \text { Hartree } \\
\text { Sum of electronic and thermal Enthalpies }=-1080.819374 \text { Hartree } \\
\text { Sum of electronic and thermal Free Energies }=-1080.888828 \text { Hartree }\end{array}$} \\
\hline Total & $\begin{array}{l}\text { rmal) } \\
\text { Mol } \\
47\end{array}$ & $\begin{array}{c}\text { CV } \\
\text { Cal/Mol-K } \\
97.761 \\
\end{array}$ & $\begin{array}{c}\mathrm{S} \\
\text { Cal/Mol-K } \\
146.179 \\
\end{array}$ & & \\
\hline \multicolumn{6}{|c|}{ Frequencies $\left(\mathrm{cm}^{-1}\right)$} \\
\hline 55.3 & 89.1 & 104.7 & 118.6 & 130.8 & 146.8 \\
\hline 174.1 & 188.7 & 189.6 & 228.7 & 234.3 & 244.9 \\
\hline 257.0 & 269.6 & 277.1 & 282.4 & 290.8 & 299.0 \\
\hline 302.3 & 321.5 & 334.0 & 339.9 & 363.8 & 374.7 \\
\hline 390.3 & 409.7 & 414.5 & 431.4 & 439.9 & 447.7 \\
\hline 481.4 & 481.6 & 492.7 & 518.0 & 527.1 & 555.8 \\
\hline 569.1 & 569.6 & 574.0 & 594.2 & 601.7 & 610.2 \\
\hline 637.6 & 656.9 & 681.7 & 690.8 & 693.2 & 705.2 \\
\hline 713.0 & 725.7 & 733.7 & 744.6 & 752.0 & 760.9 \\
\hline 767.5 & 780.2 & 783.9 & 794.5 & 796.1 & 797.4 \\
\hline 831.9 & 833.5 & 870.0 & 877.9 & 884.1 & 887.9 \\
\hline 927.6 & 943.2 & 983.1 & 1006.8 & 1027.9 & 1049.0 \\
\hline 1054.9 & 1060.4 & 1063.0 & 1070.9 & 1101.8 & 1109.7 \\
\hline 1126.4 & 1136.6 & 1154.8 & 1158.7 & 1173.8 & 1187.3 \\
\hline 1190.9 & 1216.9 & 1227.1 & 1231.1 & 1239.4 & 1246.5 \\
\hline 1255.0 & 1259.4 & 1324.1 & 1325.9 & 1334.3 & 1350.8 \\
\hline 1356.4 & 1357.9 & 1388.5 & 1400.6 & 1426.2 & 1432.2 \\
\hline 1435.0 & 1438.5 & 1438.8 & 1443.6 & 1445.8 & 1459.5 \\
\hline 1467.9 & 1497.2 & 1498.2 & 1502.0 & 1508.6 & 1515.3 \\
\hline 1518.2 & 1527.5 & 1529.2 & 1539.9 & 1545.1 & 1549.2 \\
\hline 1552.3 & 1560.1 & 1564.8 & 1594.6 & 1602.6 & 1603.2 \\
\hline 2973.2 & 2973.5 & 3012.1 & 3016.1 & 3016.5 & 3036.8 \\
\hline 3038.5 & 3063.3 & 3064.2 & 3067.5 & 3082.5 & 3082.9 \\
\hline 3098.0 & 3098.0 & 3110.5 & 3110.6 & 3117.3 & 3117.4 \\
\hline 3118.5 & 3118.7 & 3125.2 & 3126.5 & 3162.3 & 3162.4 \\
\hline
\end{tabular}




\begin{tabular}{|c|c|c|c|c|c|}
\hline \multicolumn{6}{|c|}{$\sigma$-bonded $14^{2-}$ (singlet), $C_{2}$ symmetry } \\
\hline \multicolumn{6}{|c|}{ RB3LYP/6-31G* and UB3LYP/6-31G* optimizations give the same results } \\
\hline \multicolumn{6}{|c|}{$\begin{array}{l}\text { Energy }=-1081.24927395 \text { Hartree } \\
\text { Zero-point correction }=0.426436 \text { Hartree } \\
\text { Thermal correction to Energy }=0.447960 \text { Hartree } \\
\text { Thermal correction to Enthalpy }=0.448904 \text { Hartree } \\
\text { Thermal correction to Gibbs Free Energy }=0.382152 \text { Hartree } \\
\text { Sum of electronic and zero-point Energies }=-1080.822838 \text { Hartree } \\
\text { Sum of electronic and thermal Energies }=-1080.801314 \text { Hartree } \\
\text { Sum of electronic and thermal Enthalpies }=-1080.800370 \text { Hartree } \\
\text { Sum of electronic and thermal Free Energies }=-1080.867122 \text { Hartree }\end{array}$} \\
\hline Total & $\begin{array}{l}\text { mal) } \\
\text { Mol } \\
99\end{array}$ & $\begin{array}{c}\mathrm{CV} \\
\mathrm{Cal} / \mathrm{Mol}-\mathrm{K} \\
96.692\end{array}$ & $\begin{array}{c}\mathrm{S} \\
\mathrm{Cal} / \mathrm{Mol}-\mathrm{K} \\
140.493\end{array}$ & & \\
\hline \multicolumn{6}{|c|}{ Frequencies $\left(\mathrm{cm}^{-1}\right)$} \\
\hline 88.1 & 97.6 & 128.7 & 148.1 & 159.0 & 179.4 \\
\hline 192.4 & 215.6 & 222.0 & 243.8 & 259.6 & 271.9 \\
\hline 276.4 & 286.8 & 293.4 & 317.0 & 318.8 & 333.9 \\
\hline 334.8 & 341.2 & 343.9 & 367.1 & 368.4 & 380.3 \\
\hline 402.9 & 412.8 & 429.2 & 436.6 & 449.2 & 472.5 \\
\hline 485.6 & 493.3 & 500.2 & 519.9 & 553.7 & 568.3 \\
\hline 575.1 & 584.0 & 594.7 & 596.2 & 605.9 & 617.8 \\
\hline 628.7 & 638.4 & 655.0 & 674.8 & 688.3 & 698.5 \\
\hline 700.1 & 718.4 & 730.3 & 746.6 & 755.9 & 768.1 \\
\hline 780.0 & 781.8 & 784.5 & 788.3 & 818.5 & 828.4 \\
\hline 870.5 & 876.7 & 877.6 & 892.5 & 893.4 & 893.7 \\
\hline 901.9 & 940.6 & 973.8 & 974.0 & 1047.6 & 1055.0 \\
\hline 1055.3 & 1063.2 & 1072.4 & 1076.8 & 1093.9 & 1097.8 \\
\hline 1113.5 & 1115.4 & 1147.0 & 1147.2 & 1149.6 & 1164.6 \\
\hline 1172.7 & 1188.1 & 1197.6 & 1217.4 & 1225.7 & 1240.6 \\
\hline 1242.8 & 1252.4 & 1265.1 & 1266.7 & 1301.3 & 1321.4 \\
\hline 1350.9 & 1352.2 & 1390.2 & 1410.5 & 1419.2 & 1421.4 \\
\hline 1427.8 & 1433.8 & 1435.9 & 1437.6 & 1442.3 & 1442.8 \\
\hline 1474.6 & 1487.2 & 1508.4 & 1510.7 & 1516.2 & 1517.0 \\
\hline 1531.1 & 1537.2 & 1541.8 & 1542.2 & 1548.7 & 1553.4 \\
\hline 1568.8 & 1574.3 & 1588.9 & 1596.9 & 1631.1 & 1635.4 \\
\hline 3030.2 & 3034.4 & 3034.5 & 3047.2 & 3047.4 & 3052.2 \\
\hline 3059.0 & 3061.7 & 3064.1 & 3082.4 & 3088.8 & 3089.3 \\
\hline 3112.1 & 3112.3 & 3119.2 & 3119.5 & 3123.1 & 3123.9 \\
\hline 3130.2 & 3131.2 & 3151.5 & 3151.7 & 3176.0 & 3176.1 \\
\hline
\end{tabular}

